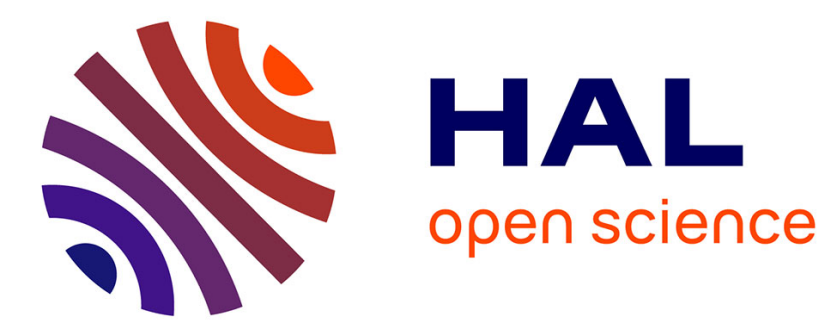

\title{
Pricing European options and risk measurement under exponential Lévy models - a practical guide
}

Khaled Salhi

\section{To cite this version:}

Khaled Salhi. Pricing European options and risk measurement under exponential Lévy models a practical guide. International Journal of Financial Engineering, 2017, 2 (2-3), 1750016 (36 p.). 10.1142/S2424786317500165 . hal-01322698v2

\section{HAL Id: hal-01322698 \\ https://hal.inria.fr/hal-01322698v2}

Submitted on 18 Nov 2016

HAL is a multi-disciplinary open access archive for the deposit and dissemination of scientific research documents, whether they are published or not. The documents may come from teaching and research institutions in France or abroad, or from public or private research centers.
L'archive ouverte pluridisciplinaire HAL, est destinée au dépôt et à la diffusion de documents scientifiques de niveau recherche, publiés ou non, émanant des établissements d'enseignement et de recherche français ou étrangers, des laboratoires publics ou privés. 


\title{
Pricing European options and risk measurement under Exponential Lévy models - a practical guide
}

\author{
Khaled Salhi $a, b, c, \dagger$
}

November 18, 2016

प

\begin{abstract}
This paper provides a thorough survey of the European option pricing, with new trends in the risk measurement, under exponential Lévy models. We develop all steps of pricing from equivalent martingale measures construction to numerical valuation of the option price under these measures. We then construct an algorithm, based on Rockafellar and Uryasev representation and fast Fourier transform, to compute Risk indicators, like the VaR and the CVaR of derivatives. The results are illustrated with an example of each exponential Lévy class. The main contribution of this paper is to build a comprehensive study from the theoretical point of view to practical numerical illustration and to give a complete characterization of the studied equivalent martingale measures by discussing their similarity and their applicability in practice. Furthermore, this work proposes applications to the Fourier inversion technique in risk measurement.
\end{abstract}

Keywords: Lévy process, incomplete market, Esscher martingale measure, minimal entropy martingale measure, fast Fourier transform, Value-at-Risk, Conditional Value-atRisk, Merton model, variance gamma model.

\section{Introduction}

Stochastic processes are intensively used for modeling financial markets. The Black and Scholes model is one of the most known models. It describes the stock price as a geometric Brownian motion. In this context, the option pricing problem is solved using the risk neutral approach [8]. The key tool is the uniqueness of the equivalent martingale measure $(\mathrm{EMM})$ and the derivative price is therefore the unique arbitrage-free contingent claim value.

It has become clear, however, that this option pricing model is inconsistent with options data. In the real world, we observe that asset price processes have jumps or spikes and risk managers have to take them into consideration. Moreover, the empirical

\footnotetext{
${ }^{a}$ Université de Lorraine, Institut Elie Cartan de Lorraine, UMR 7502, Vandoeuvre-lès-Nancy, F-54506, France.

${ }^{b}$ CNRS, Institut Elie Cartan de Lorraine, UMR 7502, Vandoeuvre-lès-Nancy, F-54506, France.

${ }^{c}$ Inria, Villers-lès-Nancy, F-54600, France.

$\dagger$ Email: Khaled.Salhi@inria.fr
} 
distributions of asset returns exhibit fat tails and skewness behaviors that deviate from normality [5]. Hence, models that accurately fit return distributions are essential to estimate profit ans loss (P\&L) distributions. Similarly, in the risk-neutral world, we observe that implied volatilities are constant neither across strikes nor across maturities as stipulated by the Black and Scholes model [39, 40]. Therefore, traders need models that can capture the behavior of the implied volatility smiles more accurately, in order to handle the risk of trades. Lévy processes provide the appropriate tools to adequately and consistently describe all these observations, both in the real world and in the risk-neutral world [4, 15, 16, 33].

By allowing the stock price process to jump, problems become more complicated. As soon as the security can have more than a single jump size, the market will be incomplete. Thus, under the assumption of no arbitrage, there are infinitely many equivalent martingale measures. This induces an interval of arbitrage-free prices. In order to construct an option pricing model, we have to select a suitable martingale measure. Once an equivalent martingale measure $\mathbb{P}^{*}$ is selected, the price $\pi(H)$ of an option $H$ is given by

$$
\pi(H)=\mathbb{E}^{*}\left[e^{-r T} H\right]
$$

where $r$ is the risk-free interest rate and $T$ is a maturity.

This survey is a practical guide to option pricing when the log of the stock price is modeled with a Lévy process. This work aims at explaining in a single document all stages of the option valuation process, as a global understanding of this process is necessary and useful for a practical purposes. Considering a price process model under the historical probability measure, we explicit this model under an equivalent martingale measure. Then, we numerically compute the option price using the fast Fourier transform technique (FFT) developed in [11].

Outline. This paper is organized as follows. In Section 2, we give an overview of the exponential Lévy model and the option pricing in this context. For background information on exponential Lévy models, the reader may refer to textbooks [2, 13]. In Section 3, we explain how to define an equivalent martingale measure $\mathbb{P}^{*}$ using the Esscher transform technique. We detail two examples: the Esscher martingale measure and the minimal entropy martingale measure. For these measures, the log of stock price process is still a Lévy process under $\mathbb{P}^{*}$ and its characteristic triplet is known. The similarity between the Esscher martingale measure and the minimal entropy martingale measure is studied. Otherwise, we show that while the former is still a good tool for pricing applications, the latter cannot be applied in a practical context. In Section 4, we give the MadanCarr method and develop an expression of the option price, based on the characteristic function of the log of price process. The application of the FFT here is possible and is the subject of Section 5. In Section 6, we develop an algorithm to compute simultaneous the VaR and the CVaR of derivatives by applying the Fourier inversion technique detailed above. Finally, in Section 7, we detail our approach on three examples of exponential Lévy models: the standard Black and Scholes model, the Merton model and the variance gamma model.

\section{Exponential Lévy model}

In this section, we introduce the exponential Lévy model and give some of its properties.

Definition 2.1. Let $\left(\Omega, \mathcal{F},\left(\mathcal{F}_{t}\right)_{t \in[0, T]}, \mathbb{P}\right)$ be a filtered probability space satisfying the usual conditions. The exponential Lévy model is defined by an asset price process $\left(S_{t}\right)_{t \in[0, T]}$ of 
the form:

$$
S_{t}=S_{0} e^{X_{t}}
$$

where $S_{0}>0$ is a constant and $\left(X_{t}\right)_{t \in[0, T]}$ is a one-dimensional Lévy process [2, [7, 43] with a characteristic triplet $\left(b, \sigma^{2}, \nu\right)$. The discounted price is given by

$$
\tilde{S}_{t}=e^{-r t} S_{t}
$$

where $r$ is the risk-free interest rate.

Notation. In the sequel, the notations used in Definition 2.1 will always be valid.

The measure $\nu$ on $\mathbb{R}$, called the Lévy measure, determines the intensity of jumps of different sizes: $\nu\left(\left[a_{1}, a_{2}\right]\right)$ is the expected number of jumps on the time interval $[0,1]$, whose sizes fall in $\left[a_{1}, a_{2}\right]$. The Lévy measure satisfies the integrability condition

$$
\int_{\mathbb{R}} 1 \wedge|x|^{2} \nu(\mathrm{d} x)<\infty
$$

Note that $\nu\left(\left[a_{1}, a_{2}\right]\right)$ is still finite for any compact set $\left[a_{1}, a_{2}\right]$ such that $0 \notin\left[a_{1}, a_{2}\right]$. If not, the process $\left(X_{t}\right)_{t \in[0, T]}$ would have an infinite number of jumps of finite size on every time interval $[0, t]$, which contradicts the càdlàg property of $\left(X_{t}\right)_{t \in[0, T]}$. Thus $\nu$ defines a Radon measure on $\mathbb{R} \backslash\{0\}$. However, $\nu$ is not necessarily a finite measure. The above restriction still allows it to blow up at zero and $\left(X_{t}\right)_{t \in[0, T]}$ may have an infinite number of small jumps on $[0, t]$. In this case, the sum of the jumps becomes an infinite series and its convergence imposes some additional conditions on the measure $\nu$.

The law of $X_{t}$ at any time $t$ is determined by the triplet $\left(b, \sigma^{2}, \nu\right)$. In particular, the Lévy-Khintchine representation gives the characteristic function of $\left(X_{t}\right)_{t \in[0, T]}$ under $\mathbb{P}$

$$
\Phi_{t}(u):=\mathbb{E}\left[e^{i u X_{t}}\right]=e^{t \Psi(u)}, \quad u \in \mathbb{R}
$$

where $\Psi$, called the characteristic exponent, is given by

$$
\Psi(u)=i b u-\frac{1}{2} \sigma^{2} u^{2}+\int_{\mathbb{R}}\left(e^{i u x}-1-i u x \mathbf{1}_{|x| \leqslant 1}\right) \nu(\mathrm{d} x) .
$$

Furthermore, if the Lévy measure also satisfies the condition $\int_{|x| \leqslant 1}|x| \nu(\mathrm{d} x)<\infty$, the jump part process, defined by

$$
X_{t}^{J}=\sum_{\substack{s \in(0, t] \\ \Delta X_{s} \neq 0}} \Delta X_{s},
$$

becomes a finite variation process. In this case, the process $\left(X_{t}\right)_{t \in[0, T]}$ can be expressed as the sum of a linear drift, a Brownian motion and a jump part process:

$$
X_{t}=\gamma t+\sigma B_{t}+X_{t}^{J}
$$

where $\gamma=b-\int_{|x| \leqslant 1} x \nu(\mathrm{d} x)$. The characteristic exponent can be expressed by

$$
\Psi(u)=i \gamma u-\frac{1}{2} \sigma^{2} u^{2}+\int_{\mathbb{R}}\left(e^{i u x}-1\right) \nu(\mathrm{d} x) .
$$

Note that the Lévy triplet of $\left(X_{t}\right)_{t \in[0, T]}$ is not given by $\left(\gamma, \sigma^{2}, \nu\right)$, but by $\left(b, \sigma^{2}, \nu\right)$. In fact, $b$ is not an intrinsic quantity and depends on the truncation function used in the 
Lévy-Khintchine representation while $\gamma$ has an intrinsic interpretation as the expectation slope of the continuous part process of $\left(X_{t}\right)_{t \in[0, T]}$. The expectation $\mathbb{E}\left[X_{t}\right]$ is given by the sum of the linear drift and the expectation of jump part equal to $\left(\gamma+\int_{\mathbb{R}} x \nu(\mathrm{d} x)\right) t$.

Now, by using Itô's formula, we can observe that $\left(S_{t}\right)_{t \in[0, T]}$ is the solution to the following SDE

$$
S_{t}=S_{0}+\int_{(0, t]} S_{s-} d \hat{X}_{s}
$$

where

$$
\hat{X}_{t}:=X_{t}+\frac{1}{2}\left\langle X^{c}\right\rangle_{t}+\sum_{s \in(0, t]}\left\{e^{\Delta X_{s}}-1-\Delta X_{s}\right\}
$$

and $\left(X_{t}^{c}\right)_{t \in[0, T]}$ is the continuous part of $\left(X_{t}\right)_{t \in[0, T]}$. Hence, $\left(S_{t}\right)_{t \in[0, T]}$ can be rewritten as:

$$
S_{t}=S_{0} \mathcal{E}(\hat{X})_{t}
$$

where $\left(\mathcal{E}(\hat{X})_{t}\right)_{t \in[0, T]}$ stands for the Doléans-Dade exponential of $\left(\hat{X}_{t}\right)_{t \in[0, T]}[28]$. Furthermore, $\left(\hat{X}_{t}\right)_{t \in[0, T]}$ is still a Lévy process under $\mathbb{P}$. By expressing its Lévy-Itô decomposition, we obtain that the characteristic triplet of $\left(\hat{X}_{t}\right)_{t \in[0, T]}$ is given by $\left(\hat{b}, \sigma^{2}, \hat{\nu}\right)$ (see [13, 20] for details) where

$$
\hat{b}=b+\frac{1}{2} \sigma^{2}+\int_{|x| \leqslant 1} x \hat{\nu}(\mathrm{d} x)-\int_{|x| \leqslant 1} x \nu(\mathrm{d} x)
$$

and

$$
\hat{\nu}(\mathrm{d} x)=\nu \circ J^{-1}(\mathrm{~d} x) \text { where } J(x):=e^{x}-1 \text { for } x \in \mathbb{R} .
$$

Remark 2.1. $\quad$ (i) It holds that

$$
\operatorname{supp}\{\hat{\nu}\} \subset(-1, \infty)
$$

(ii) If $\nu$ has a density $\nu(x)$, then $\hat{\nu}$ has a density $\hat{\nu}(x)$ given by

$$
\hat{\nu}(x)=\frac{1}{1+x} \nu(\log (1+x)) .
$$

(iii) From the economical point of view, $\left(X_{t}\right)_{t \in[0, T]}$ represents the logarithmic return process of $\left(S_{t}\right)_{t \in[0, T]}$, while $\left(\hat{X}_{t}\right)_{t \in[0, T]}$ represents the simple return process of $\left(S_{t}\right)_{t \in[0, T]}$.

We consider a call with maturity $T$ and strike $K$. The payoff of this option is given by the random variable $H=\left(S_{T}-K\right)_{+}$. Let $\mathcal{P}$ denote the set of all equivalent martingale measures (also called risk-neutral measures)

$$
\mathcal{P}=\left\{\mathbb{P}^{*} \sim \mathbb{P}, \quad\left(\tilde{S}_{t}\right)_{t \in[0, T]} \text { is a martingale under } \mathbb{P}^{*}\right\}
$$

where $\left(\tilde{S}_{t}\right)_{t \in[0, T]}$ is given by $(2)$.

In a complete market, there is only one equivalent martingale measure $\mathbb{P}^{*}$ and the risk-neutral price of the option at $t=0$ is given by

$$
C(K)=e^{-r T} \mathbb{E}^{*}\left[\left(S_{T}-K\right)_{+}\right]
$$

where $\mathbb{E}^{*}$ is the expectation under $\mathbb{P}^{*}$. 
With exponential Lévy models, we are mostly in the incomplete market case. Therefore, several equivalent martingale measures can be used to price the option. The range of option prices is given by

$$
\left[\inf _{\mathbb{P} * \mathcal{P}} e^{-r T} \mathbb{E}^{*}\left[\left(S_{T}-K\right)_{+}\right], \sup _{\mathbb{P} * \mathcal{P}} e^{-r T} \mathbb{E}^{*}\left[\left(S_{T}-K\right)_{+}\right]\right] .
$$

So, one can always choose a measure $\mathbb{P}^{*} \in \mathcal{P}$ according to some criteria and price the option using the formula (5).

Another difficulty with exponential Lévy models is that closed-form expressions exist for their characteristic function while their density function is usually unknown. It is thus difficult to find a closed-form formula of $C(K)$, and even not possible for some pricing measures and Lévy processes. Nevertheless, the analytic expression of the characteristic function $\Phi_{t}^{*}$ under the pricing measure $\mathbb{P}^{*}$ is known, one can use the fast Fourier transform (FFT) method developed by Carr and Madan [11 to numerically compute the option price.

Assume now that the characteristic function $\Phi_{t}$ of $\left(X_{t}\right)_{t \in[0, T]}$ under $\mathbb{P}$ is analytically known. The pricing procedure has two steps:

1. Choose an equivalent martingale measure $\mathbb{P}^{*} \in \mathcal{P}$ under which we have an analytic expression of the characteristic function, called $\Phi_{t}^{*}$.

2. Apply the FFT in $\Phi_{T}^{*}$ to compute the option price.

\section{Equivalent martingale measure}

The equivalent martingale measure method is one of the most powerful methods of option pricing. The no-arbitrage assumption can be expressed by the existence of at least one equivalent martingale measure. If the market is arbitrage-free and incomplete, there are several equivalent martingale measures and we have to select, with respect to some criteria, the most suitable one in order to price options.

Several candidates for an equivalent martingale measure are proposed in the literature. To construct them, two different approaches are employed:

- Esscher transform method: The Esscher transform method is widely used in risk theory. It consists in applying an Esscher transform with respect to some risk process. This risk process can be the logarithmic return $\left(X_{t}\right)_{t \in[0, T]}$ in the case of the Esscher martingale measure [9, 22], the simple return $\left(\hat{X}_{t}\right)_{t \in[0, T]}$ in the case of the Minimal entropy martingale measure [19, 20, 35] or the continuous martingale part $\left(X_{t}^{c}\right)_{t \in[0, T]}$ of the Lévy process $\left(X_{t}\right)_{t \in[0, T]}$ in the case of the mean correcting martingale measure [49].

- Minimal distance method: This method is more related to the maximization of expected utility and hedging problem. This includes the utility-based martingale measure [27], the minimal martingale measure [18] and the variance optimal martingale measure [44].

\subsection{Esscher martingale measure}

The Esscher martingale measure is constructed by applying an Esscher transform with respect to the process $\left(X_{t}\right)_{t \in[0, T]}$. One of the greatest advantages is that $\left(X_{t}\right)_{t \in[0, T]}$ is still a Lévy process under this equivalent measure. Let us give the definition of the Esscher transform and the condition under which we obtain an equivalent martingale measure. 
Definition 3.1. Let $\left(X_{t}\right)_{t \in[0, T]}$ be a Lévy process on $\left(\Omega, \mathcal{F},\left(\mathcal{F}_{t}\right)_{t \in[0, T]}, \mathbb{P}\right)$. We call Esscher transform with respect to $\left(X_{t}\right)_{t \in[0, T]}$ any change of $\mathbb{P}$ to an equivalent measure $\mathbb{P}^{*}$ by a density process $Z_{t}=\left.\frac{\mathrm{d} \mathbb{P}^{*}}{\mathrm{~d} \mathbb{P}}\right|_{\mathcal{F}_{t}}$ of the form:

$$
Z_{t}=\frac{e^{\theta X_{t}}}{\mathbb{E}\left[e^{\theta X_{t}}\right]},
$$

where $\theta \in \mathbb{R}$.

The Esscher density process $Z_{t}=\left.\frac{\mathrm{d} \mathbb{P} *}{\mathrm{~d} \mathbb{P}}\right|_{\mathcal{F}_{t}}$, which formally looks like the density of a one-dimensional Esscher transform, leads to one-dimensional Esscher transforms of the marginal distributions, with the same parameter $\theta$ :

$$
\mathbb{P}^{*}\left(X_{t} \in B\right)=\int \mathbf{1}_{B}\left(X_{t}\right) \frac{e^{\theta X_{t}}}{\mathbb{E}\left[e^{\theta X_{t}}\right]} \mathrm{d} \mathbb{P}=\frac{1}{\mathbb{E}\left[e^{\theta X_{t}}\right]} \int \mathbf{1}_{B}(x) e^{\theta x} \mathrm{~d} \mathbb{P}^{X_{t}}(\mathrm{~d} x)
$$

for any set $B \in \mathcal{B}(\mathbb{R})$.

One advantage of using $\left(X_{t}\right)_{t \in[0, T]}$ as a risk process is that the density process only depends on the current stock price. In what follows, we give the condition for the existence of the density process $Z_{t}=\left.\frac{d \mathbb{P}^{*}}{d \mathbb{P}}\right|_{\mathcal{F}_{t}}$ and the characteristic triplet of $\left(X_{t}\right)_{t \in[0, T]}$ under $\mathbb{P}^{*}$ in such case.

Proposition 3.1. Let $\left(X_{t}\right)_{t \in[0, T]}$ be a Lévy process on $\mathbb{R}$ with characteristic triplet $\left(b, \sigma^{2}, \nu\right)$ and let $\theta \in \mathbb{R}$. The exponential moment $\mathbb{E}\left[e^{\theta X_{t}}\right]$ is finite for some $t$ or, equivalently, for all $t>0$ if and only if $\int_{|x| \geqslant 1} e^{\theta x} \nu(\mathrm{d} x)<\infty$. In this case,

$$
\mathbb{E}\left[e^{\theta X_{t}}\right]=e^{t \Psi(-i \theta)},
$$

where $\Psi$ is the characteristic exponent of the Lévy process defined by (3).

For a proof, see [43, Theorem 25.17].

Proposition 3.2. Let $\left(X_{t}\right)_{t \in[0, T]}$ be a Lévy process on $\mathbb{R}$ with characteristic triplet $\left(b, \sigma^{2}, \nu\right)$ under $\mathbb{P}$. For all $\theta \in \mathbb{R}$ such that $\mathbb{E}\left[e^{\theta X_{1}}\right]<\infty$,

(i) the process $\left(Z_{t}\right)_{t \in[0, T]}$ given by (6) defines a density process;

(ii) the process $\left(X_{t}\right)_{t \in[0, T]}$ is a Lévy process with triplet $\left(b^{*}, \sigma^{2}, \nu^{*}\right)$ under $\mathbb{P}^{*}$ where

$$
\nu^{*}(\mathrm{~d} x)=e^{\theta x} \nu(\mathrm{d} x), \quad \text { for } x \in \mathbb{R}
$$

and

$$
b^{*}=b+\sigma^{2} \theta+\int_{|x| \leqslant 1} x \nu^{*}(\mathrm{~d} x)-\int_{|x| \leqslant 1} x \nu(\mathrm{d} x) .
$$

Proof. (i) Recall that $\mathbb{E}\left[e^{\theta X_{t}}\right]=e^{t \Psi(-i \theta)}=\left(\mathbb{E}\left[e^{\theta X_{1}}\right]\right)^{t}$. Then, $Z_{t}$ is integrable for all $t$. Using the independence and stationary properties of the Lévy process $\left(X_{t}\right)_{t \in[0, T]}$, we have for $s<t$,

$$
\begin{aligned}
\mathbb{E}\left[Z_{t} \mid \mathcal{F}_{s}\right] & =\frac{1}{\mathbb{E}\left[e^{\theta X_{t}}\right]} \mathbb{E}\left[e^{\theta\left(X_{t}-X_{s}+X_{s}\right)} \mid \mathcal{F}_{s}\right]=\frac{1}{\mathbb{E}\left[e^{\theta X_{t}}\right]} \mathbb{E}\left[e^{\theta\left(X_{t}-X_{s}\right)} \mid \mathcal{F}_{s}\right] \mathbb{E}\left[e^{\theta X_{s}} \mid \mathcal{F}_{s}\right] \\
& =\frac{1}{\mathbb{E}\left[e^{\theta X_{t}}\right]} \mathbb{E}\left[e^{\theta\left(X_{t}-X_{s}\right)}\right] e^{\theta X_{s}}=\frac{e^{\theta X_{s}}}{\mathbb{E}\left[e^{\theta X_{s}}\right]}=Z_{s}
\end{aligned}
$$


Thus, $\left(Z_{t}\right)_{t \in[0, T]}$ is a $\mathbb{P}$-martingale.

(ii) We prove that $\left(X_{t}\right)_{t \in[0, T]}$ is a Lévy process under the probability measure $\mathbb{P}^{*}$ by computing its characteristic function under $\mathbb{P}^{*}$ :

$$
\begin{aligned}
\Phi^{*}(u) & =\mathbb{E}^{*}\left[e^{i u X_{t}}\right]=\int e^{i u X_{t}} \mathrm{~d} \mathbb{P}^{*}=\int e^{i u X_{t}} \frac{e^{\theta X_{t}}}{\mathbb{E}\left[e^{\theta X_{t}}\right]} \mathrm{d} \mathbb{P} \\
& =\frac{\mathbb{E}\left[e^{(\theta+i u) X_{t}}\right]}{\mathbb{E}\left[e^{\theta X_{t}}\right]}=\exp (t(\Psi(-i(\theta+i u))-\Psi(-i \theta))) .
\end{aligned}
$$

Define $\Psi^{*}(u) \equiv \Psi(-i(\theta+i u))-\Psi(-i \theta)$ for $u \in \mathbb{R}$. Then,

$$
\begin{aligned}
\Psi^{*}(u)= & \left(b(\theta+i u)+\frac{\sigma^{2}}{2}(\theta+i u)^{2}+\int_{\mathbb{R}}\left(e^{(\theta+i u) x}-1-(\theta+i u) x \mathbf{1}_{|x| \leqslant 1}\right) \nu(\mathrm{d} x)\right) \\
& -\left(b \theta+\frac{\sigma^{2}}{2} \theta^{2}+\int_{\mathbb{R}}\left(e^{\theta x}-1-\theta x \mathbf{1}_{|x| \leqslant 1}\right) \nu(\mathrm{d} x)\right) \\
= & i\left(b+\sigma^{2} \theta\right) u-\frac{\sigma^{2}}{2} u^{2}+\int_{\mathbb{R}}\left(e^{\theta x}\left(e^{i u x}-1\right)-i u x \mathbf{1}_{|x| \leqslant 1}\right) \nu(\mathrm{d} x) \\
= & i\left(b+\sigma^{2} \theta+\int_{|x| \leqslant 1}\left(e^{\theta x}-1\right) x \nu(\mathrm{d} x)\right) u-\frac{\sigma^{2}}{2} u^{2}+\int_{\mathbb{R}}\left(e^{\theta x}\left(e^{i u x}-1-i u x \mathbf{1}_{|x| \leqslant 1}\right)\right) \nu(\mathrm{d} x) .
\end{aligned}
$$

By defining

$$
\nu^{*}(x)=e^{\theta x} \nu(x), \text { for } x \in \mathbb{R}
$$

and

$$
b^{*}=b+\sigma^{2} \theta+\int_{|x| \leqslant 1} x \nu^{*}(\mathrm{~d} x)-\int_{|x| \leqslant 1} x \nu(\mathrm{d} x),
$$

we obtain a Lévy-Khintchine representation for $\Phi^{*}$. Thus, $\left(X_{t}\right)_{t \in[0, T]}$ is a Lévy process under $\mathbb{P}^{*}$ with triplet $\left(b^{*}, \sigma^{2}, \nu^{*}\right)$.

To interpret the expression of $b^{*}$, let us remember that the jump measure $\nu$ may have a singularity at zero. Thus, there can be infinitely many small jumps and the characteristic function of their sum $\int_{|x| \leqslant 1}\left(e^{i u x}-1\right) \nu(\mathrm{d} x)$ does not necessarily converge. To obtain convergence, this jump integral was centered and replaced by its compensated version in the Lévy-Khintchine representation. We integrate this compensator $\int_{|x| \leqslant 1} x \nu(\mathrm{d} x)$ in the drift. When we change the measure $\mathbb{P}$ to $\mathbb{P}^{*}$, we must naturally truncate the compensator of $\nu$ from the drift and add the one of $\nu^{*}$. We thus obtain $b^{*}$.

Theorem 3.1. Let $\left(X_{t}\right)_{t \in[0, T]}$ be a Lévy process with triplet $\left(b, \sigma^{2}, \nu\right)$ under $\mathbb{P}$. Suppose that $X_{1}$ is non-degenerate and has a moment generating function $u \mapsto \mathbb{E}\left[\exp \left(u X_{1}\right)\right]$ on some open interval $\left(a_{1}, a_{2}\right)$ with $a_{2}-a_{1}>1$. Assume that there exists a real number $\theta \in\left(a_{1}, a_{2}-1\right)$ such that

$$
b+\sigma^{2} \theta+\frac{\sigma^{2}}{2}+\int_{\mathbb{R}}\left(e^{\theta x}\left(e^{x}-1\right)-x \mathbf{1}_{|x| \leqslant 1}\right) \nu(\mathrm{d} x)=r,
$$

or equivalently

$$
\mathbb{E}\left[e^{(\theta+1) X_{t}}\right]=e^{r t} \mathbb{E}\left[e^{\theta X_{t}}\right],
$$

where $r$ is the risk-free interest rate. Then the real $\theta$ is unique and the equivalent measure $\mathbb{P}^{*}$ given by the Esscher transform with respect to $\left(X_{t}\right)_{t \in[0, T]}$

$$
\left.\frac{\mathrm{d} \mathbb{P}^{*}}{\mathrm{~d} \mathbb{P}}\right|_{\mathcal{F}_{t}}=\frac{e^{\theta X_{t}}}{\mathbb{E}\left[e^{\theta X_{t}}\right]}
$$

is an equivalent martingale measure. 
Proof. Proposition 3.2 guarantees that $\left(X_{t}\right)_{t \in[0, T]}$ is a Lévy process under all measures $\mathbb{P}^{*}$ given by an Esscher transform. By the independence and stationarity of increments of $\left(X_{t}\right)_{t \in[0, T]}$, the martingale property of $\left(S_{t}\right)_{t \in[0, T]}$ under $\mathbb{P}^{*}$ is implied by $\mathbb{E}^{*}\left[\tilde{S}_{t}\right]=S_{0}$ for $t>0$. From the definition of the Esscher measure transform,

$$
\mathbb{E}^{*}\left[\tilde{S}_{t}\right]=\mathbb{E}^{*}\left[S_{0} e^{X_{t}-r t}\right]=\mathbb{E}\left[S_{0} e^{X_{t}-r t} \frac{e^{\theta X_{t}}}{\mathbb{E}\left[e^{\theta X_{t}}\right]}\right]=S_{0} e^{-r t} \frac{\mathbb{E}\left[e^{(\theta+1) X_{t}}\right]}{\mathbb{E}\left[e^{\theta X_{t}}\right]} .
$$

Thus, $\mathbb{E}^{*}\left[\tilde{S}_{t}\right]=S_{0}$ if and only if there exists a real $\theta$ such that $(8)$ holds. This real $\theta$ must be in $\left(a_{1}, a_{2}-1\right)$ to ensure the existence of the moment generating function in $\theta$ and $\theta+1$.

Using Proposition 3.1, we rewrite (8) in terms of the characteristic exponent under $\mathbb{P}$

$$
\Psi(-i(\theta+1))-\Psi(-i \theta)=r .
$$

We then develop the expression of $\Psi$ given by (3) and we obtain the condition (7). The discounted price $\left(\tilde{S}_{t}\right)_{t \in[0, T]}$ is a martingale under the equivalent measure given by the Esscher transform with $\theta$ (if it exists) solution to this equation (7).

\subsection{Minimal Entropy martingale measure (MEMM)}

The MEMM has been investigated in various settings by several authors [17, 18, 19, 35, 44. In particular, the MEMM for Exponential Lévy process has been discussed in [12, 20, 24, 36]. It turns out that this measure can be obtained by applying an Esscher transform with respect to the simple return process $\left(\hat{X}_{t}\right)_{t \in[0, T]}$. Furthermore, $\left(X_{t}\right)_{t \in[0, T]}$ is still a Lévy process under this measure. In this section, we recall the definition of the relative entropy and give the condition on the Esscher parameter for the existence of the MEMM, as well as the characteristic triplet of $\left(X_{t}\right)_{t \in[0, T]}$ under this measure.

Definition 3.2. Let $\mathcal{G}$ be a sub- $\sigma$-field of $\mathcal{F}$ and $\mathbb{Q}$ a probability measure on $\mathcal{G}$. The relative entropy on $\mathcal{G}$ of $\mathbb{Q}$ with respect to $\mathbb{P}$ is defined by

$$
\mathbb{H}_{\mathcal{G}}(\mathbb{Q} \mid \mathbb{P}):= \begin{cases}\int \log \left(\left.\frac{\mathrm{d} \mathbb{Q}}{\mathrm{d} \mathbb{P}}\right|_{\mathcal{G}}\right) \mathrm{d} \mathbb{Q}, & \text { if } \mathbb{Q} \ll \mathbb{P} \text { on } \mathcal{G}, \\ +\infty, & \text { otherwise }\end{cases}
$$

where $\left.\frac{\mathrm{d} \mathbb{Q}}{\mathrm{d}}\right|_{\mathcal{G}}$ stands for the Radon-Nikodym derivative of $\left.\mathbb{Q}\right|_{\mathcal{G}}$ with respect to $\left.\mathbb{P}\right|_{\mathcal{G}}$.

Theorem 3.2. Let $\left(X_{t}\right)_{t \in[0, T]}$ be a Lévy process with triplet $\left(b, \sigma^{2}, \nu\right)$ under $\mathbb{P}$. Suppose that there exists a real number $\beta \in \mathbb{R}$ such that

$$
\int_{x>1} e^{x} e^{\beta\left(e^{x}-1\right)} \nu(\mathrm{d} x)<\infty
$$

and

$$
b+\sigma^{2} \beta+\frac{\sigma^{2}}{2}+\int_{\mathbb{R}}\left(\left(e^{x}-1\right) e^{\beta\left(e^{x}-1\right)}-x \mathbf{1}_{|x| \leqslant 1}\right) \nu(\mathrm{d} x)=r,
$$

where $r$ is the risk-free interest rate. Then,

1. The real $\beta$ is unique and the equivalent measure $\mathbb{P}^{\dagger}$ given by the Esscher transform with respect to $\left(\hat{X}_{t}\right)_{t \in[0, T]}$,

$$
\left.\frac{\mathrm{d} \mathbb{P}^{\dagger}}{\mathrm{d} \mathbb{P}}\right|_{\mathcal{F}_{t}}=\frac{e^{\beta \hat{X}_{t}}}{\mathbb{E}\left[e^{\beta \hat{X}_{t}}\right]}
$$

is an equivalent martingale measure, where $\left(\hat{X}_{t}\right)_{t \in[0, T]}$ is given by (4). 
2. The stochastic process $\left(X_{t}\right)_{t \in[0, T]}$ is still a Lévy process under $\mathbb{P}^{\dagger}$ with characteristic triplet $\left(b^{\dagger}, \sigma^{2}, \nu^{\dagger}\right)$ where

$$
b^{\dagger}=b+\beta \sigma^{2}+\int_{|x| \leqslant 1} x \nu^{\dagger}(\mathrm{d} x)-\int_{|x| \leqslant 1} x \nu(\mathrm{d} x)
$$

and

$$
\nu^{\dagger}(\mathrm{d} x)=e^{\beta\left(e^{x}-1\right)} \nu(\mathrm{d} x)
$$

3. The probability measure $\mathbb{P}^{\dagger}$ attains the minimal entropy in $\mathcal{P}$

$$
\mathbb{H}_{\mathcal{F}_{T}}\left(\mathbb{P}^{\dagger} \mid \mathbb{P}\right)=\min _{\mathbb{Q} \in \mathcal{P}} \mathbb{H}_{\mathcal{F}_{T}}(\mathbb{Q} \mid \mathbb{P})
$$

For a proof, see [20].

Thus, the minimal entropy martingale measure can be simply expressed as an Esscher transform with respect to the simple return process $\left(\hat{X}_{t}\right)_{t \in[0, T]}$. Note that although we have the characteristic triplet of the process $\left(X_{t}\right)_{t \in[0, T]}$ under $\mathbb{P}^{\dagger}$, the analytic expression of the characteristic function under this equivalent martingale measure is often difficult to express and the pricing with the characteristic function is not possible in this case.

\section{Pricing with characteristic function}

We consider here the problem of European call valuation of maturity $T$. Various techniques have been applied to answer this question. For example, one can resort to Monte Carlo techniques to simulate sample paths for the asset. Averaging a sufficiently large number of realized payoffs yields the required price, see for example [6, 23]. One can also attempt to derive a partial differential equation for pricing which can be solved using numerical methods [48]. Yet another method is based on the Fourier analysis, which is the subject of the current section.

Two methods based on the Fourier analysis exist in the literature. Both of them rely on the availability of the characteristic function of the log of the stock price. Indeed, for a wide class of stock models characteristic functions have been obtained in a closed-form formula even if the risk-neutral densities (or probability mass function) themselves are not explicitly available. Examples of characteristic functions of Lévy process have been derived in [25, 32, 50].

The first of these Fourier methods is actually the application of the Gil-Palaez inversion formula in finance. This idea originates from [25]. However, singularities in the integrand prevent it to be an accurate method. The second, called the Carr-Madan method, was first proposed by [11]. It ensures that the Fourier transform of the call price exists thanks to the inclusion of a damping factor. Moreover, the Fourier inversion can be accomplished by the fast Fourier transform (FFT) in this case. The tremendous speed of the FFT allows option pricing for a huge number of strikes to be evaluated very rapidly. In this section, we illustrate the Carr-Madan method.

Let $S_{T}=S_{0} \exp \left(X_{T}\right)$ be the terminal price of the underlying asset of a European call with strike $K$, where $\left(X_{t}\right)_{t \in[0, T]}$ is a Lévy process with triplet $\left(b, \sigma^{2}, \nu\right)$. Denote by $\mathbb{P}^{*}$ the selected equivalent martingale measure and by $f_{T}^{*}$ (its analytic expression is unknown) the risk-neutral density of $X_{T}$. The characteristic function of $X_{T}$ under $\mathbb{P}^{*}$ can be written as

$$
\Phi_{T}^{*}(u)=\int_{\mathbb{R}} e^{i u x} f_{T}^{*}(x) \mathrm{d} x .
$$


Let $k=\log \left(K / S_{0}\right)$ be the $\log$ of the normalized strike. The risk-neutral valuation under $\mathbb{P}^{*}$ yields

$$
\begin{aligned}
C(K) & =e^{-r T} \mathbb{E}^{*}\left[\left(S_{T}-K\right)_{+}\right]=S_{0} e^{-r T} \mathbb{E}^{*}\left[\left(e^{X_{T}}-e^{k}\right)_{+}\right] \\
& =S_{0} e^{-r T} \int_{k}^{\infty}\left(e^{x}-e^{k}\right) f_{T}^{*}(x) \mathrm{d} x .
\end{aligned}
$$

Define the function $c_{0}$ by $c_{0}(k)=C\left(S_{0} e^{k}\right)$. The Fourier inversion technique consists in the following assertion: $C(K)=c_{0}(k)$ and $c_{0}=\mathrm{FT}^{-1} \circ \mathrm{FT}\left(c_{0}\right)$ where $\mathrm{FT}$ is the Fourier transform operator. Since

$$
\lim _{k \rightarrow-\infty} c_{0}(k)=\lim _{K \rightarrow 0} C(K)=S_{0}
$$

we see that $c_{0}$ is not in $L^{1}$, the space of integrable functions, as the limit of $c_{0}(k)$ as $k$ goes to minus infinity is different from zero. For that, we cannot directly apply the Fourier inversion technique as the Fourier transform of $c_{0}(k)$ does not converge. To get around this problem of integrability, we consider the modified call price

$$
c_{\alpha}(k)=e^{\alpha k} c_{0}(k),
$$

where $\alpha>0$.

In the next two propositions, we develop a closed-form formula for the Fourier transform of $c_{\alpha}(k)$ and obtain the option price $C(K)$ by applying the inverse Fourier transform to the developed formula.

Proposition 4.1. Let $\alpha>0$ such that $\mathbb{E}^{*}\left[e^{(\alpha+1) X_{T}}\right]<\infty$. The Fourier transform of $c_{\alpha}(k)$ is well defined and given by:

$$
\hat{c}_{\alpha}(v)=\frac{S_{0} e^{-r T} \Phi_{T}^{*}(v-(\alpha+1) i)}{\alpha^{2}+\alpha-v^{2}+i(2 \alpha+1) v}, \quad \forall v \in \mathbb{R}
$$

where $\Phi_{T}^{*}$ is the characteristic function of $X_{T}$ under $\mathbb{P}^{*}$.

Proof. Assume for the moment that $\hat{c}_{\alpha}(v)$ is well defined. We have

$$
\begin{aligned}
\hat{c}_{\alpha}(v) & =\int_{-\infty}^{\infty} e^{i v k} c_{\alpha}(k) \mathrm{d} k=\int_{-\infty}^{\infty} e^{i v k} e^{\alpha k} C\left(S_{0} e^{k}\right) \mathrm{d} k \\
& =\int_{-\infty}^{\infty} e^{i v k} e^{\alpha k}\left(S_{0} e^{-r T} \int_{k}^{\infty}\left(e^{x}-e^{k}\right) f_{T}^{*}(x) \mathrm{d} x\right) \mathrm{d} k \\
& =S_{0} e^{-r T} \int_{-\infty}^{\infty} f_{T}^{*}(x)\left(\int_{-\infty}^{x} e^{(\alpha+i v) k}\left(e^{x}-e^{k}\right) \mathrm{d} k\right) \mathrm{d} x \\
& =S_{0} e^{-r T} \int_{-\infty}^{\infty} f_{T}^{*}(x)\left(e^{x} \int_{-\infty}^{x} e^{(\alpha+i v) k} \mathrm{~d} k-\int_{-\infty}^{x} e^{(\alpha+1+i v) k} \mathrm{~d} k\right) \mathrm{d} x \\
& =S_{0} e^{-r T} \int_{-\infty}^{\infty} f_{T}^{*}(x)\left(\frac{e^{(\alpha+1+i v) x}}{\alpha+i v}-\frac{e^{(\alpha+1+i v) x}}{\alpha+1+i v}\right) \mathrm{d} x .
\end{aligned}
$$

By substituting (9) here in, we obtain the expression (10).

We now prove the existence of $\hat{c}_{\alpha}(v)$. First note that $\mathbb{E}^{*}\left[e^{(\alpha+1) X_{T}}\right]<\infty$ implies

$$
\hat{c}_{\alpha}(0)<\infty
$$


since

$$
\hat{c}_{\alpha}(0)=\frac{S_{0} e^{-r T} \Phi_{T}^{*}(-(\alpha+1) i)}{\alpha^{2}+\alpha}=\frac{S_{0} e^{-r T} \mathbb{E}^{*}\left[e^{(\alpha+1) X_{T}}\right]}{\alpha^{2}+\alpha} .
$$

On the other hand, as $c_{\alpha}(k)$ is positive, we have

$$
\left|\hat{c}_{\alpha}(v)\right|=\left|\int_{-\infty}^{\infty} e^{i v k} c_{\alpha}(k) \mathrm{d} k\right| \leqslant \int_{-\infty}^{\infty} c_{\alpha}(k) \mathrm{d} k=\hat{c}_{\alpha}(0) .
$$

Combining this with (11) completes the proof.

Proposition 4.2. Let $\left(X_{t}\right)_{t \in[0, T]}$ be a Lévy process with characteristic function $\Phi^{*}$ under an equivalent martingale measure $\mathbb{P}^{*}$. The option price is given by

$$
C(K)=\frac{e^{-\alpha \log \left(K / S_{0}\right)}}{\pi} \operatorname{Re}\left\{\int_{0}^{\infty} e^{-i v \log \left(K / S_{0}\right)} \hat{c}_{\alpha}(v) \mathrm{d} v\right\},
$$

where $\hat{c}_{\alpha}$ is given by 10 .

Proof. The inverse Fourier transform gives us

$$
c_{\alpha}(k)=\frac{1}{2 \pi} \int_{\mathbb{R}} e^{-i v k} \hat{c}_{\alpha}(v) \mathrm{d} v .
$$

Then,

$C(K)=\frac{e^{-\alpha \log \left(K / S_{0}\right)}}{2 \pi} \int_{\mathbb{R}} e^{-i v \log \left(K / S_{0}\right)} \hat{c}_{\alpha}(v) \mathrm{d} v=\frac{e^{-\alpha \log \left(K / S_{0}\right)}}{\pi} \operatorname{Re}\left\{\int_{0}^{\infty} e^{-i v \log \left(K / S_{0}\right)} \hat{c}_{\alpha}(v) \mathrm{d} v\right\}$

where the last equality follows from the observation that

$$
\int_{\mathbb{R}} e^{-i v \log \left(K / S_{0}\right)} \hat{c}_{\alpha}(v) \mathrm{d} v=\int_{0}^{\infty} e^{-i v \log \left(K / S_{0}\right)} \hat{c}_{\alpha}(v) \mathrm{d} v+\int_{-\infty}^{0} e^{-i v \log \left(K / S_{0}\right)} \hat{c}_{\alpha}(v) \mathrm{d} v,
$$

and where the second term on the right-hand side can be written as

$$
\begin{aligned}
\int_{-\infty}^{0} e^{-i v \log \left(K / S_{0}\right)} \hat{c}_{\alpha}(v) \mathrm{d} v & =\frac{\int_{0}^{\infty} e^{i u \log \left(K / S_{0}\right)} \hat{c}_{\alpha}(-u) \mathrm{d} u}{\int_{0}^{\infty} e^{-i u \log \left(K / S_{0}\right)} \hat{c}_{\alpha}(u) \mathrm{d} u} .
\end{aligned}
$$

This concludes the proof.

We only have considered the pricing of vanilla calls. Obviously, one can obtain prices of vanilla puts by using the put-call parity. The price $P_{T}(K)$ of a vanilla put can alternatively be obtained with the Carr-Madan inversion by choosing a negative value for $\alpha$, see [31].

\section{Discretization and FFT}

Computing the price of a call option $C(K)=e^{-r T} \mathbb{E}^{*}\left[\left(S_{T}-K\right)_{+}\right]$under the pricing rule $\mathbb{P}^{*}$ requires the inversion of the Fourier transform in 12 . In general, this will not be analytically tractable. A numerical approach is necessary. In doing so we give a 
formulation to which we can apply the fast Fourier transform (FFT) [14, 47]. Here we define the discrete Fourier transform (DFT) as

$$
F_{u}=\sum_{n=1}^{N} f_{n} \omega_{N}^{(n-1)(u-1)}, \quad u=1, \ldots, N
$$

where $\omega_{N}=e^{-\frac{2 \pi i}{N}}$. The software R provides an efficient FFT-algorithm for this formulation.

We are interested in computing the integral

$$
\int_{0}^{\infty} e^{-i v k} \hat{c}_{\alpha}(v) \mathrm{d} v
$$

where $\hat{c}_{\alpha}(v)$ is given by $(10)$.

For $g_{k}(v) \equiv e^{-i v k} \hat{c}_{\alpha}(v)$, the trapezoidal rule yields

$$
\begin{aligned}
\int_{0}^{A} g_{k}(v) \mathrm{d} v & \approx \frac{\Delta v}{2}\left[g_{k}\left(v_{1}\right)+2 \sum_{n=2}^{N-1} g_{k}\left(v_{n}\right)+g_{k}\left(v_{N}\right)\right] \\
& =\Delta v\left[\sum_{n=1}^{N} g_{k}\left(v_{n}\right)-\frac{1}{2}\left[g_{k}\left(v_{1}\right)+g_{k}\left(v_{N}\right)\right]\right]
\end{aligned}
$$

where $A=(N-1) \Delta v$. As we truncated the interval of integration, a truncation error will result and we refer to [11] for discussions on this topic. Let

$$
v_{n}=(n-1) \Delta v, \quad n=1, \ldots, N .
$$

Furthermore, let

$$
k_{u}=k_{1}+(u-1) \Delta k, \quad u=1, \ldots, N .
$$

be the grid in the $k$-domain. The constant $k_{1} \in \mathbb{R}$ can be tuned such that the grid is laid around aimed strikes. If we are interested in options with particular strikes around a value $K$, we take $k_{1}=\log \left(K / S_{0}\right)-\frac{N}{2} \Delta v$. Substituting (15) and (16) in (14) yields

$$
\begin{aligned}
\int_{0}^{A} g_{k_{u}}(v) \mathrm{d} v & \approx \Delta v\left[\sum_{n=1}^{N} e^{-i[(n-1) \Delta v]\left[k_{1}+(u-1) \Delta k\right]} \hat{c}_{\alpha}\left(v_{n}\right)-\frac{1}{2}\left[g_{k_{u}}\left(v_{1}\right)+g_{k_{u}}\left(v_{N}\right)\right]\right] \\
& =\Delta v\left[\sum_{n=1}^{N} e^{-i \Delta v \Delta k(n-1)(u-1)} g_{k_{1}}\left(v_{n}\right)-\frac{1}{2}\left[g_{k_{u}}\left(v_{1}\right)+g_{k_{u}}\left(v_{N}\right)\right]\right]
\end{aligned}
$$

By setting

$$
\Delta v \Delta k=\frac{2 \pi}{N}
$$

we have

$$
\int_{0}^{A} g_{k_{u}}(v) \mathrm{d} v \approx \Delta v\left[\sum_{n=1}^{N} \omega_{N}^{(n-1)(u-1)} g_{k_{1}}\left(v_{n}\right)-\frac{1}{2}\left[g_{k_{u}}\left(v_{1}\right)+g_{k_{u}}\left(v_{N}\right)\right]\right] .
$$

The sum in (17) takes the form of (13) with $f_{n}=g_{k_{1}}\left(v_{n}\right)$. Hence FFT can be applied to evaluate it. The final result for the Carr-Madan inversion is thus:

$$
\left.C\left(S_{0} e^{k_{u}}\right) \approx \frac{e^{-\alpha k_{u}}}{\pi} \operatorname{Re}\left\{\Delta v\left[\sum_{n=1}^{N} \omega_{N}^{(n-1)(u-1)} g_{k_{1}}\left(v_{n}\right)\right]-\frac{1}{2}\left[g_{k_{u}}\left(v_{1}\right)+g_{k_{u}}\left(v_{N}\right)\right]\right]\right\} .
$$


Instead of the trapezoidal rule, we can apply the more accurate Simpson's rule. Along the same lines as above, one can easily show that in this case we have

$$
\begin{array}{r}
C\left(S_{0} e^{k_{u}}\right) \approx \frac{e^{-\alpha k_{u}}}{\pi} \operatorname{Re}\left\{\frac { \Delta v } { 3 } \left[\sum_{n=1}^{N} \omega_{N}^{(n-1)(u-1)} g_{k_{1}}\left(v_{n}\right)\left(3+(-1)^{n}-\delta_{n-1}\right)\right.\right. \\
\left.\left.-\left[g_{k_{u}}\left(v_{N-1}\right)+4 g_{k_{u}}\left(v_{N}\right)\right]\right]\right\},
\end{array}
$$

where $\delta_{j-1}$ denotes the Kronecker delta function that equals 1 whenever $j=1$.

To apply the FFT algorithm, $N$ must be a power of 2 . For that, we fix $N=4096$ and $\Delta v=0.25$. This gives $\Delta k=6.13 \cdot 10^{-3}$. We are interested in strikes around at the money $K=S_{0}$. We fix then $k_{1}=-\frac{N}{2} \Delta k$.

\section{Risk measurement of derivatives}

In this section, we propose a method to compute the risk of derivatives based on Fourier inversion technique explained above. Two well known indicators to measure the risk are the Value-at-Risk (VaR) [29] and the Conditional Value-at-Risk (CVaR) [1]. The VaR of the payoff is the maximum potential value of the payoff that can occur with a given level of confidence. In short,

$$
\mathbb{P}\left(H \leqslant \operatorname{VaR}_{a}(H)\right)=a,
$$

where $a$ is the confidence level. As the VaR only gives a payoff threshold, it was completed by the CVaR which is defined by

$$
\operatorname{CVaR}_{a}(H)=\frac{1}{1-a} \int_{a}^{1} \operatorname{VaR}_{x}(H) \mathrm{d} x=\mathbb{E}\left[H \mid H>\operatorname{VaR}_{a}(H)\right]
$$

Rockafellar and Uryasev [37, 38] found an equivalent formula for CVaR as a convex function, thus opening the door for convex programming methods. They proved that the CVaR is equivalent to

$$
\mathrm{CVaR}_{a}(H)=\min _{z \in \mathbb{R}}\left\{z+\frac{1}{1-a} \mathbb{E}\left[(H-z)_{+}\right]\right\},
$$

and that $\operatorname{VaR}_{a}(H)$ is the leftpoint of the argmin set of this function.

Consider a European call option with payoff $H=\left(S_{T}-K\right)_{+}$. As $H$ is always positive, $\operatorname{VaR}_{a}(H)$ and $\mathrm{CVaR}_{a}(H)$ are also positive. Then,

$$
\begin{aligned}
\operatorname{CVaR}_{a}(H) & =\min _{z \geqslant 0}\left\{z+\frac{1}{1-a} \mathbb{E}\left[(H-z)_{+}\right]\right\} \\
& =\min _{z \geqslant 0}\left\{z+\frac{1}{1-a} \mathbb{E}\left[\left(\left(S_{T}-K\right)_{+}-z\right)_{+}\right]\right\} \\
& =\min _{z \geqslant 0}\left\{z+\frac{1}{1-a} \mathbb{E}\left[\left(S_{T}-(K+z)\right)_{+}\right]\right\} .
\end{aligned}
$$

For all $z \geqslant 0$, the quantity $\mathbb{E}\left[\left(S_{T}-(K+z)\right)_{+}\right]$takes the form of option pricing formula with strike $K+z$ under the probability measure $\mathbb{P}$. These quantities can be computed by fast Fourier transform, as explained in Sections 4 and 5, since we know the characteristic function $\Phi_{T}$ of $X_{T}$ under $\mathbb{P}$. We minimize then on the grid of $z$, to obtain the VaR and the CVaR. The method is described by the following algorithm: 


$$
\begin{aligned}
& \text { Fix } N \text { and } \Delta v . \\
& \text { Using Fourier inversion technique, compute } \mathbb{E}\left[\left(S_{T}-K_{u}\right)_{+}\right], u=1, \cdots, N . \\
& \text { Define } z_{u}=K_{u}-K \text { for } K_{u} \geqslant K . \\
& \mathrm{CVaR}_{a} \approx \min _{z_{u}}\left\{z_{u}+\frac{1}{1-a} \mathbb{E}\left[\left(S_{T}-K_{u}\right)_{+}\right]\right\} \text {and } \operatorname{VaR}_{a} \approx \underset{z_{u}}{\operatorname{argmin}}\left\{z_{u}+\frac{1}{1-a} \mathbb{E}\left[\left(S_{T}-K_{u}\right)_{+}\right]\right\} .
\end{aligned}
$$

Algorithm 1: $\operatorname{VaR}_{a}\left(\left(S_{t}-K\right)_{+}\right)$and $\operatorname{CVaR}_{a}\left(\left(S_{t}-K\right)_{+}\right)$computing.

Similar reasoning, that combines the Rockafellar and Uryasev algorithm and the Fourier inversion technique, permits to compute different risk indicators in the exponentialLévy context, as the monetary Value-at-Risk and Conditional Value-at-Risk $\left(\operatorname{VaR}_{a}\left(S_{0}-\right.\right.$ $\left.S_{T}\right)$ and $\left.\operatorname{CVaR}_{a}\left(S_{0}-S_{T}\right)\right)$ and the Value-at-Risk and Conditional Value-at-Risk in terms of returns $\left(\operatorname{VaR}_{a}\left(-X_{T}\right)\right.$ and $\left.\mathrm{CVaR}_{a}\left(-X_{T}\right)\right)$ [42].

\section{Applications}

Financial models with jumps fall into two categories. In the first category, called jumpdiffusion models, the evolution of prices is given by a diffusion process, punctuated by jumps at random intervals. Here the jumps represent rare events as crashes and large drawdowns. Such an evolution can be represented by modeling the log of the price as a Lévy process with a nonzero Gaussian component and a jump part, which is a compound Poisson process with a finite number of jumps in every time interval. Examples of such models are the Merton jumps diffusion model with Gaussian jumps [34 and the Kou model with double exponential jumps [30]. In these models, the dynamical structure of the process is easy to understand and to describe, since the distribution of jump sizes is known. The second category consists of models with an infinite number of jumps in every time interval, which are called infinite activity models. In these models, one does not need to introduce a Brownian component since the dynamics of jumps is already rich enough to generate a nontrivial small time behavior [10] and it has been argued [10, 21] that such models give a more realistic description of the price process at various time scales. In addition, many models from this class can be constructed via Brownian subordination which gives them additional analytic tractability compared to jump-diffusion models. Two important examples of this category are the variance gamma model [11, 32] and the normal inverse Gaussian model [3, 4, 41]. However, since the real price process is observed on a discrete grid, it is difficult, and even impossible, to empirically observe to which category the price process belongs. The choice becomes rather a question of modeling convenience than an empirical one. In this section, we start by recalling the standard Black and Scholes model. Then, we apply the pricing method in a jump-diffusion example, the Merton model. We end by the variance gamma model: an infinite activity example. Unless otherwise stated, for numerical applications, we use the parameters summarized in Table 1

\subsection{Black and Scholes model}

The Black and Scholes model, proposed by [8, is one of the most popular models in finance. The price process $\left(S_{t}\right)_{t \in[0, T]}$ is solution to the SDE

$$
d S_{t}=\mu S_{t} d t+\sigma S_{t} d B_{t} ; S_{0}>0
$$




\begin{tabular}{rrrrr}
\hline Market & B\&S & Merton & VG & FFT \\
\hline$S_{0}=100$ & $\mu=0.145$ & $\gamma=0.1$ & $\gamma=0.1$ & $N=4096$ \\
$r=0.02$ & $\sigma=0.3$ & $\sigma=0.3$ & $m=-0.01$ & $\Delta v=0.25$ \\
$T=0.5$ & & $\lambda=1$ & $\delta=1$ & $\Delta k=\frac{2 \pi}{N \Delta v}$ \\
& & $m=-0.1$ & $\kappa=0.2$ & $k_{1}=-\frac{N}{2} \Delta k$ \\
& & & \\
\hline
\end{tabular}

Table 1: Summary of numerical values of different parameters used in Section 6.

where $\mu \in \mathbb{R}, \sigma>0$ and $\left(B_{t}\right)_{t \in[0, T]}$ is a standard Brownian motion. By applying Itô's formula, we re-write $\left(S_{t}\right)_{t \in[0, T]}$ as an exponential Lévy process (1) where

$$
X_{t}=\left(\mu-\frac{\sigma^{2}}{2}\right) t+\sigma B_{t}
$$

for $t \in[0, T]$. The process $\left(X_{t}\right)_{t \in[0, T]}$ is a Lévy process with triplet $\left(\mu-\frac{\sigma^{2}}{2}, \sigma^{2}, 0\right)$. In fact, for each $t \in[0, T]$, as $X_{t}$ follows a Gaussian distribution $\mathcal{N}\left(\left(\mu-\frac{\sigma^{2}}{2}\right) t, \sigma^{2} t\right)$, its characteristic function is given by

$$
\Phi_{t}(u)=\mathbb{E}\left[e^{i u X_{t}}\right]=\exp \left(t\left(i\left(\mu-\frac{\sigma^{2}}{2}\right) u-\frac{\sigma^{2}}{2} u^{2}\right)\right) .
$$

In this model, the price process does not have jumps. So, we are here in the complete market case where there is only one equivalent martingale measure.

Esscher martingale measure. The exponential moment $\mathbb{E}\left[e^{\theta X_{1}}\right]$ is finite for all $\theta \in \mathbb{R}$. To prove the existence of the Esscher martingale measure $\mathbb{P}^{*}$, we look for a real $\theta \in \mathbb{R}$ solution to

$$
\left(\mu-\frac{\sigma^{2}}{2}\right)+\sigma^{2} \theta+\frac{\sigma^{2}}{2}=r .
$$

The solution is given by $\theta=(r-\mu) / \sigma^{2}$ and the measure $\mathbb{P}^{*}$ defined by the Esscher transform with respect to $\left(X_{t}\right)_{t \in[0, T]}$ and with the parameter $\theta$ is the unique equivalent martingale measure. The process $\left(X_{t}\right)_{t \in[0, T]}$ is again a Lévy process under $\mathbb{P}^{*}$ with triplet $\left(r-\frac{\sigma^{2}}{2}, \sigma, 0\right)$.

This means that $\left(X_{t}\right)_{t \in[0, T]}$ can be written as

$$
X_{t}=\left(r-\frac{\sigma^{2}}{2}\right) t+\sigma W_{t}
$$

where $\left(W_{t}\right)_{t \in[0, T]}$ given by $W_{t}=B_{t}+\frac{\mu-r}{\sigma} t$ is a standard Brownian motion under $\mathbb{P}^{*}$. The discounted price

$$
\tilde{S}_{t}=S_{0} e^{\sigma W_{t}-\frac{\sigma^{2}}{2} t}
$$

solves the equation

$$
d \tilde{S}_{t}=\tilde{S}_{t} d W_{t} ; \quad S_{0}>0 .
$$

We found the same context of Black and Scholes modeling and the Girsanov theorem for measure changes. 


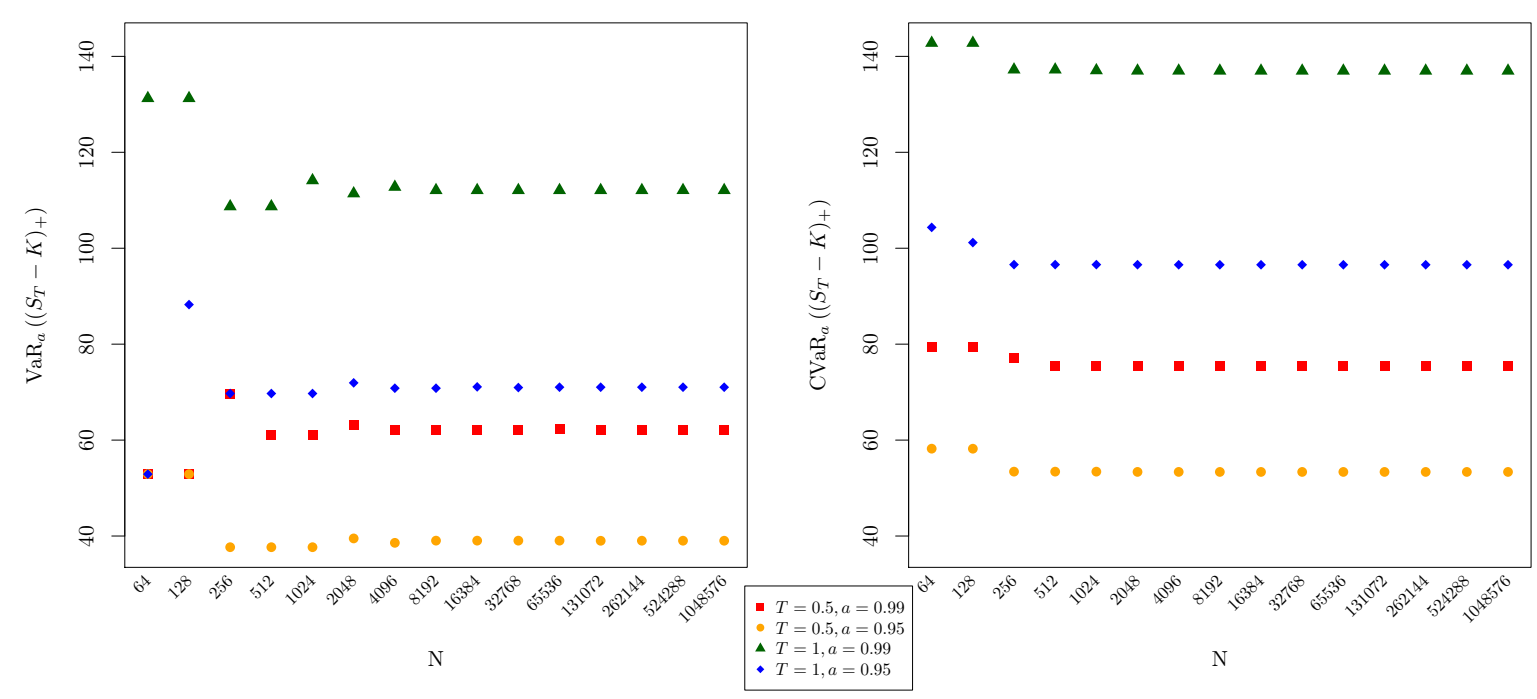

Figure 1: $\operatorname{VaR}_{a}\left(\left(S_{T}-K\right)_{+}\right)$and $\operatorname{CVaR}_{a}\left(\left(S_{T}-K\right)_{+}\right)$with respect to $N$ under Black and Scholes model.

Pricing with FFT. For a Black and Scholes model with parameters corresponding to the values in Table 1, the Esscher parameter that gives the Esscher martingale measure is $\theta=(r-\mu) / \sigma^{2}=-1.39$. The numerical computation of the call price is presented in Figure 5 with respect to the strike $K$. We see that the option price approaches $S_{0}$ as $K \rightarrow 0$ and goes to 0 as $K \rightarrow+\infty$.

Otherwise, we have compared the closed-form price of Black and Scholes with the option price given by the Carr-Madan method. The absolute error of the numerical method is of order $6 \cdot 10^{-7}$ whatever the strike is.

Risk measurement. In this paragraph, we compute the VaR and the CVaR of the call payoff under a Black and Scholes model. We take the corresponding parameters from Table 1. The strike is fixed at $K=110$. The application of the Fourier inversion gives $\mathbb{E}\left[\left(S_{T}-(K+z)\right)_{+}\right]$for a grid of $\left(K_{u}\right)_{1 \leqslant u \leqslant N}$ with steps $\Delta K_{u}=S_{0} \exp \left(k_{1}+(u-\right.$ 1) $\Delta k)-S_{0} \exp \left(k_{1}+(u-2) \Delta k\right)$ for $u=2, \ldots, N$. Thus, we obtain evaluations of the objective function of Rockafellar and Uryasev algorithm for a grid $z_{u}=K_{u}-K$ for $u=1, \ldots, N$. The minimum of the objective function and the corresponding argmin in the $z$-grid represent respectively approximations of the CVaR and the VaR.

We study the sensitivity of the VaR/CVaR computing with respect to the size of the discretization grid, for different values of time to expiration $T$ and confidence level $a$. The larger $N$ is, the smaller the steps $\Delta z_{u}$ are, which means more evaluations of the objective function. Figure 1 shows the $\mathrm{VaR}$ and the CVaR for different values of $N=2^{n}$, with $n$ in $6, \ldots, 20$. The absolute variation of the $\mathrm{VaR}$ and the CVaR values with respect to $N$ is given in Figure 2. We conclude that the $\operatorname{VaR}_{a}(H)$ is stable from $N=16384$ and that $\mathrm{CVaR}_{a}(H)$ is stable from $N=8192$. The CVaR converges faster because it is a minimum, while the $\mathrm{VaR}$ is an argmin. The CPU computing time of the method is illustrated in Figure 3. Up to $N=16384$, the algorithm behaves as instantaneous. 


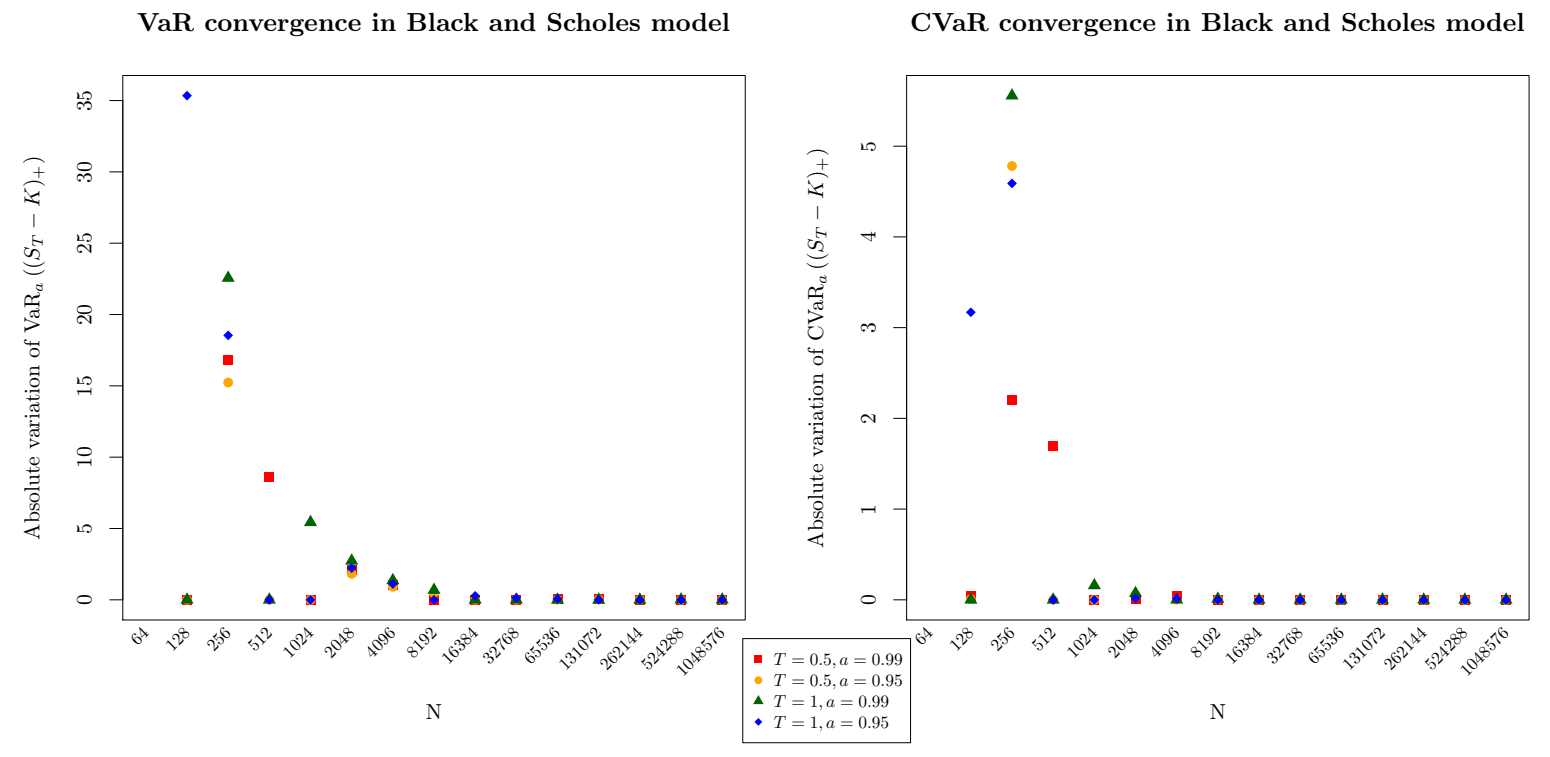

Figure 2: The absolute variation of $\operatorname{VaR}_{a}\left(\left(S_{T}-K\right)_{+}\right)$and $\mathrm{CVaR}_{a}\left(\left(S_{T}-K\right)_{+}\right)$with respect to $N$ under Black and Scholes model.

Computing time of VaR/CVaR in Black and Scholes model

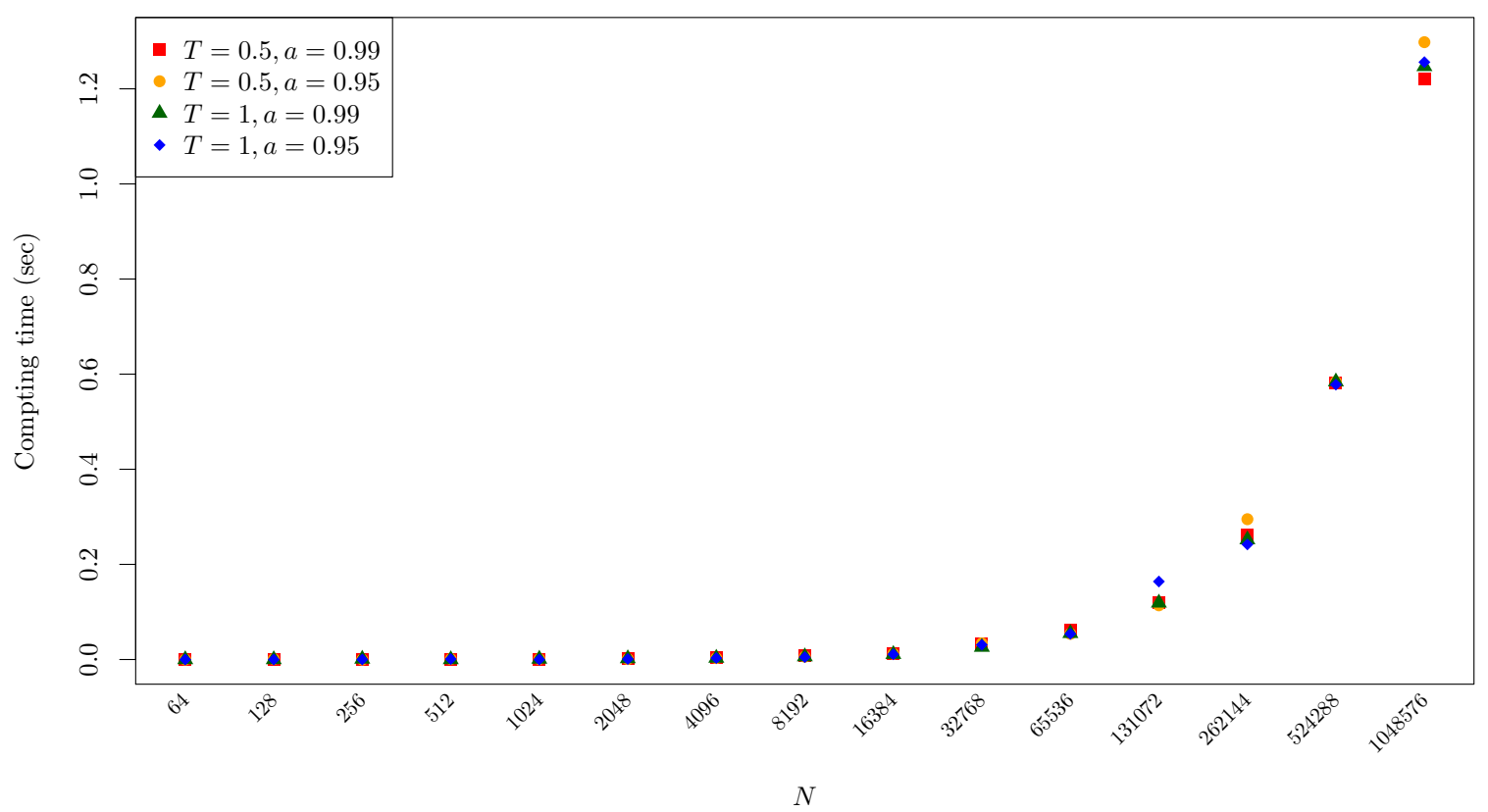

Figure 3: CPU time of the VaR/CVaR computing method under Black and Scholes model. 


\subsection{Merton model}

This model is proposed by [34]. The price is described by an exponential Lévy process (1) where

$$
X_{t}=\gamma t+\sigma B_{t}+\sum_{i=1}^{N_{t}} Y_{i},
$$

with $\gamma \in \mathbb{R},\left(B_{t}\right)_{t \in[0, T]}$ is a standard Brownian motion, $\left(N_{t}\right)_{t \in[0, T]}$ is a Poisson process with intensity $\lambda$ and $\left(Y_{i}\right)_{i \geqslant 1}$ are i.i.d. Gaussian random variables with parameters $m$ and $\delta^{2}$. For each $0 \leqslant t \leqslant T$, the characteristic function of $X$ is given by

$$
\Phi_{t}(u)=\mathbb{E}\left[e^{i u X_{t}}\right]=e^{t \Psi(u)},
$$

and

$$
\Psi(u)=i \gamma u-\frac{\sigma^{2}}{2} u^{2}+\lambda\left(\exp \left(i m u-\frac{\delta^{2}}{2} u^{2}\right)-1\right)
$$

Define

$$
\nu(x)=\lambda \times \frac{1}{\sqrt{2 \pi} \delta} \exp \left(-\frac{(x-m)^{2}}{2 \delta^{2}}\right), x \in \mathbb{R}
$$

and

$$
b=\gamma+\int_{|x| \leqslant 1} x \nu(\mathrm{d} x) .
$$

Then, $\Psi$ can be written on the form

$$
\begin{aligned}
\Psi(u) & =i \gamma u-\frac{\sigma^{2}}{2} u^{2}+\int_{\mathbb{R}}\left(e^{i u x}-1\right) \nu(\mathrm{d} x) \\
& =i b u-\frac{\sigma^{2}}{2} u^{2}+\int_{\mathbb{R}}\left(e^{i u x}-1-i u x \mathbf{1}_{|x| \leqslant 1}\right) \nu(\mathrm{d} x) .
\end{aligned}
$$

We conclude that, under $\mathbb{P},\left(X_{t}\right)_{t \in[0, T]}$ is a Lévy process with triplet $\left(b, \sigma^{2}, \nu\right)$. With this model, the market is incomplete. The compound Poisson process makes the risk uncontrolled and there exists an infinity of equivalent martingale measures. Estimation of different parameters from financial data is detailed in [46].

Esscher martingale measure. The exponential moment $\mathbb{E}\left[e^{\theta X_{1}}\right]$ is finite for all $\theta \in \mathbb{R}$. To prove the existence of the Esscher martingale measure $\mathbb{P}^{*}$, we look for a real $\theta \in \mathbb{R}$ solution to

$$
\gamma+\sigma^{2} \theta+\frac{\sigma^{2}}{2}+\int_{\mathbb{R}} e^{\theta x}\left(e^{x}-1\right) \nu(\mathrm{d} x)=r
$$

or farther

$$
\gamma+\sigma^{2} \theta+\frac{\sigma^{2}}{2}+\lambda\left(e^{m(\theta+1)+\frac{\delta^{2}}{2}(\theta+1)^{2}}-e^{m \theta+\frac{\delta^{2}}{2} \theta^{2}}\right)=r .
$$

For numerical application with the parameters given in Table 1 , we obtain $\theta \approx-0.352$.

The characteristic function of $\left(X_{t}\right)_{t \in[0, T]}$ under $\mathbb{P}^{*}$ is given by

$$
\Phi_{t}^{*}(u)=e^{t \Psi^{*}(u)},
$$

where

$$
\Psi^{*}(u)=i\left(\gamma+\sigma^{2} \theta\right) u-\frac{\sigma^{2}}{2} u^{2}+\lambda\left(e^{m(\theta+i u)+\frac{\delta^{2}}{2}(\theta+i u)^{2}}-e^{m \theta+\frac{\delta^{2}}{2} \theta^{2}}\right)
$$


We conclude that, with the Esscher martingale measure, we are able to provide analytic expression of the characteristic function under an equivalent martingale measure. This analytic form will be numerically inverted later with the fast Fourier transform to provide the option price.

Using Proposition 2.1, the process $\left(X_{t}\right)_{t \in[0, T]}$ is a Lévy process under $\mathbb{P}^{*}$ with triplet $\left(b^{*}, \sigma^{2}, \nu^{*}\right)$ defined by

$$
b^{*}=\gamma+\sigma^{2} \theta+\int_{|x| \leqslant 1} x \nu^{*}(\mathrm{~d} x)
$$

and

$$
\nu^{*}(x)=e^{\theta x} \nu(x)=\lambda^{*} \times \frac{1}{\sqrt{2 \pi} \delta} \exp \left(x-\left(m+\delta^{2} \theta\right)\right)^{2} /\left(2 \delta^{2}\right),
$$

where $\lambda^{*}=\lambda \exp \left(m \theta+\frac{\delta^{2} \theta^{2}}{2}\right)$. Hence, the Esscher martingale model $\left(X_{t}\right)_{t \in[0, T]}$ is once again a jump diffusion with compound Poisson jumps:

$$
X_{t}=\left(\gamma+\sigma^{2} \theta\right) t+\sigma B_{t}^{*}+\sum_{i=1}^{N_{t}^{*}} Y_{i}^{*}
$$

where $\left(B_{t}^{*}\right)_{t \in[0, T]}$ is a $\mathbb{P}^{*}$-standard Brownian motion, $\left(N_{t}^{*}\right)_{t \in[0, T]}$ is a $\mathbb{P}^{*}$-Poisson process with intensity $\lambda^{*}$ and $\left(Y_{i}^{*}\right)_{i \geqslant 1}$ are i.i.d. Gaussian random variables with parameters $m+\delta^{2} \theta$ and $\delta^{2}$.

Minimal entropy martingale measure. The existence of the minimal entropy martingale measure $\mathbb{P}^{\dagger}$ is equivalent to the existence of a real $\beta$ solution to

$$
\gamma+\sigma^{2} \beta+\frac{\sigma^{2}}{2}+\int_{\mathbb{R}}\left(\left(e^{x}-1\right) e^{\beta\left(e^{x}-1\right)}\right) \nu(\mathrm{d} x)=r
$$

in the Merton model framework. With the same parameters of Table1, we get numerically $\beta \approx-0.365$. by

Using Theorem 3.2. $\left(X_{t}\right)_{t \in[0, T]}$ is a Lévy process under $\mathbb{P}^{\dagger}$ with triplet $\left(b^{\dagger}, \sigma^{2}, \nu^{\dagger}\right)$ defined

$$
b^{\dagger}=\gamma+\sigma^{2} \beta+\int_{|x| \leqslant 1} x \nu^{\dagger}(\mathrm{d} x)
$$

and

$$
\nu^{\dagger}(x)=e^{\beta\left(e^{x}-1\right)} \nu(x)=\lambda^{\dagger} \rho^{\dagger}(x)
$$

where $\lambda^{\dagger}=\int \nu^{\dagger}(\mathrm{d} x)$ and $\rho^{\dagger}(x)=\frac{\nu^{\dagger}(x)}{\int \nu^{\dagger}(\mathrm{d} x)}$. Hence, the minimal entropy martingale model $\left(X_{t}\right)_{t \in[0, T]}$ is once again a jump diffusion with compound Poisson jumps:

$$
X_{t}=\left(\gamma+\sigma^{2} \beta\right) t+\sigma B_{t}^{\dagger}+\sum_{i=1}^{N_{t}^{\dagger}} Y_{i}^{\dagger}
$$

where $\left(B_{t}^{\dagger}\right)_{t \in[0, T]}$ is a $\mathbb{P}^{\dagger}$-standard Brownian motion, $\left(N_{t}^{\dagger}\right)_{t \in[0, T]}$ is a $\mathbb{P}^{\dagger}$-Poisson process with intensity $\lambda^{\dagger}$ and $\left(Y_{i}^{\dagger}\right)_{i \geqslant 1}$ are i.i.d. random variables with probability density function $\rho^{\dagger}$.

The role of the damping factor $e^{\theta x}$ in the case of the Esscher martingale measure $\mathbb{P}^{*}$ and the dumping factor $e^{\beta\left(e^{x}-1\right)}$ in the case of the minimal entropy martingale measure $\mathbb{P}^{\dagger}$ is to mitigate the general trend of the log price. In the Merton model, the general trend is given by $\mathbb{E}\left[X_{t}\right]=(\gamma+\lambda m) t$. A positive expectation slope of the log price leads to negative 
parameters $\theta$ and $\beta$ which permit to give more weight to negative jumps and less weight to positive jumps in the Lévy densities $\nu^{*}$ and $\nu^{\dagger}$. This rebalances the market in the riskneutral world and gives the martingale property. Conversely, if the log price expectation is negative, the dumping factors will strongly reduce the left tail of the risk-neutral Lévy densities.

The initial Lévy density $\nu$ and the two risk-neutral Lévy densities $\nu^{*}$ (under the Esscher martingale measure $\mathbb{P}^{*}$ ) and $\nu^{\dagger}$ (under the minimal entropy martingale measure $\mathbb{P}^{\dagger}$ ), as well as the corresponding jump densities, are depicted in Figure 4 for different values of the $\log$ price expectation. We vary the jump size expectation $m=\{-0.1,-0.3,0.3\}$ to have different signs of $\mathbb{E}\left[X_{t}\right]$ and therefore different signs of $\theta$ and $\beta$. The remaining parameters of the model are the ones given in Table 1. Depending on the Esscher parameter sign, we have a dumping of the right tail or the left tail of the risk-neutral Lévy density. Despite this dumping effect in the Lévy densities, we observe in the right hand figures that the jump size densities are not affected by this change of measures and the main effect is then an increase or decrease in the jump intensities $\lambda^{*}$ and $\lambda^{\dagger}$.

On the other hand, when we compare $\nu^{*}$ to $\nu^{\dagger}$, we find that both functions are almost equal with variations that do not exceed $1.6 \times 10^{-2}$. We can explain this by the fact that $X$ and $\hat{X}$ represent respectively the compound and the simple returns of the stock price. So, we consider models with small jump $x$ for which $e^{x}-1 \approx x$. Thus, the Esscher martingale measure and the minimal entropy martingale measure are almost the same.

While we have the triplet of $\left(X_{t}\right)_{t \in[0, T]}$ under the minimal entropy martingale measure, we still do not have an analytic expression of the characteristic function under this measure. For that, using the FFT to compute option prices under the minimal entropy martingale measure will be not possible.

Pricing with FFT under the Esscher martingale measure. In Figure 5, we compare the option price under a Black and Scholes model and a Merton model having the same expectation of $\left(X_{t}\right)_{t \in[0, T]}$. We choose models parameters such that $\mu-\frac{\sigma^{2}}{2}=\gamma+\lambda m$. The numerical computation of the call price with respect to the strike $K$, is presented in Figure 5. As the Merton model allows bigger negative jumps, the probability to finish in-the-money is smaller. Otherwise, the option price can always be written

$$
C(K)=S_{0} \Pi_{1}-K e^{-r T} \mathbb{P}\left(S_{T}>K\right),
$$

where $\Pi_{1}$ is the delta of the option. The call price is thus greater around the money in the Merton model.

Option price sensitivity to jumps. To discuss the effects of jumps on the option price, we compare the results of the Merton model with respect to the jump intensity. Recall that the Black and Scholes model and the Merton model are exponential Lévy models with triplets $\left(\mu-\frac{\sigma^{2}}{2}, \sigma^{2}, 0\right)$ and $\left(\gamma+\int_{|x| \leqslant 1} x \nu(\mathrm{d} x), \sigma^{2}, \nu\right)$ respectively, where

$$
\nu(x)=\lambda \times \frac{1}{\sqrt{2 \pi} \delta} \exp \left((x-m)^{2} /\left(2 \delta^{2}\right)\right) .
$$

Then, a Black and Scholes model is a Merton model with particular parameters $\gamma=$ $\mu-\frac{\sigma^{2}}{2}$ and $\lambda=0$. This model does not contain jumps as the jump intensity $\lambda=0$. To see the effect of jumps on the option prices, we fix parameters such that $\gamma=\mu-\frac{\sigma^{2}}{2}$ and compare the Black and Scholes model to Merton models with different jump intensities. We take the parameters of Table 1 except for $\lambda$ that varies in $\{2,4,6\}$. Note that this choice of parameters respects the condition $\gamma=\mu-\frac{\sigma^{2}}{2}$. The different option prices are 


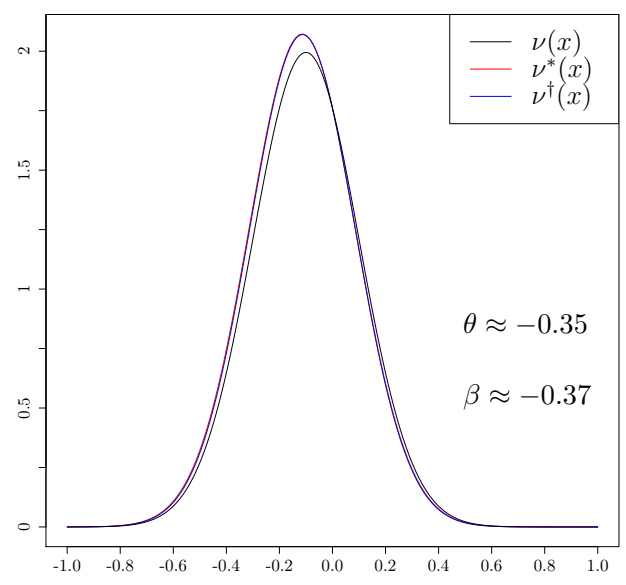

(a) $m=-0.1$

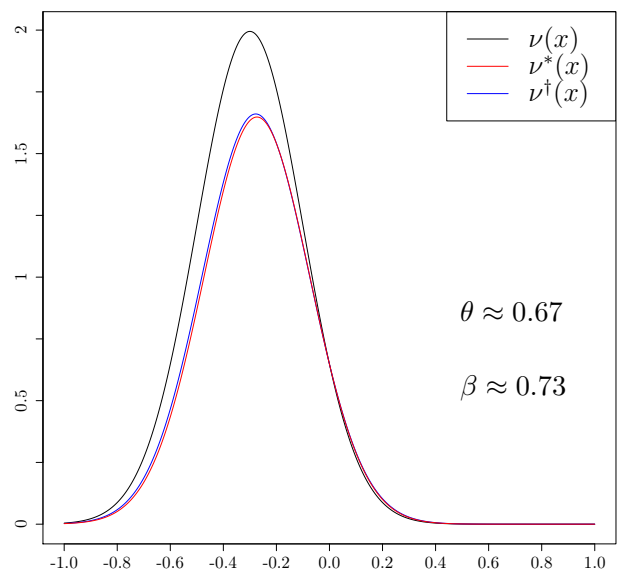

(c) $m=-0.3$

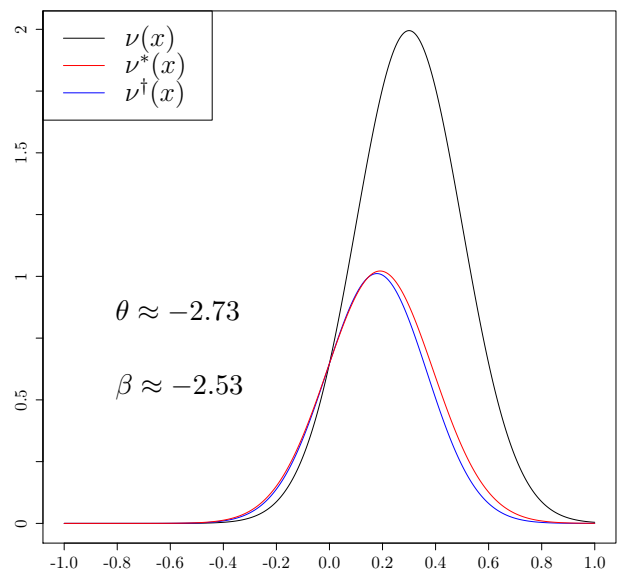

(e) $m=0.3$

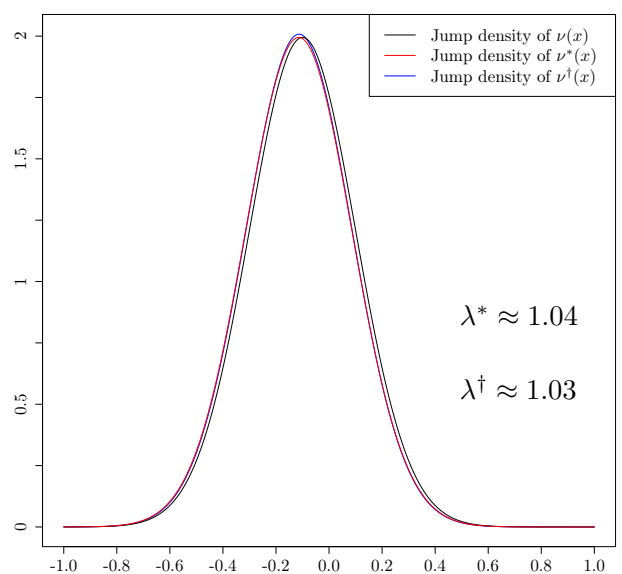

(b) $m=-0.1$

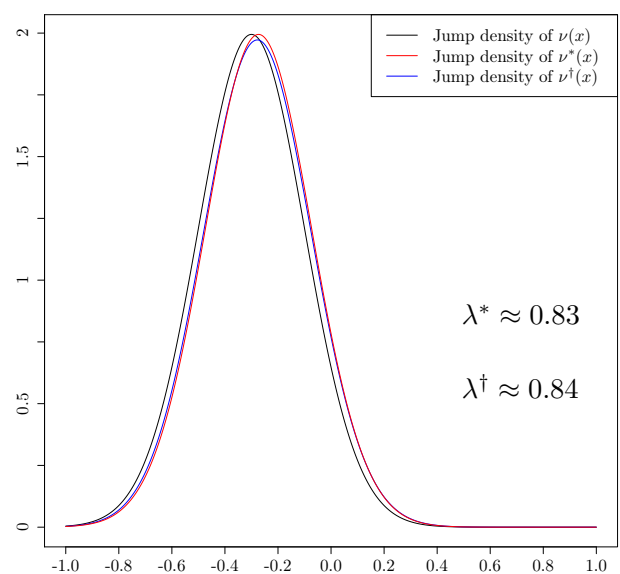

(d) $m=-0.3$

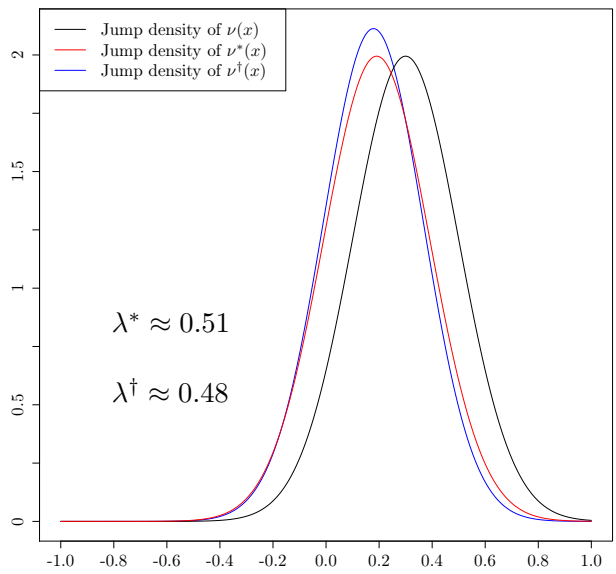

(f) $m=0.3$

Figure 4: Lévy densities and jump size densities under the historical measure, the Esscher martingale measure and the minimal entropy martingale measure in the Merton model. 


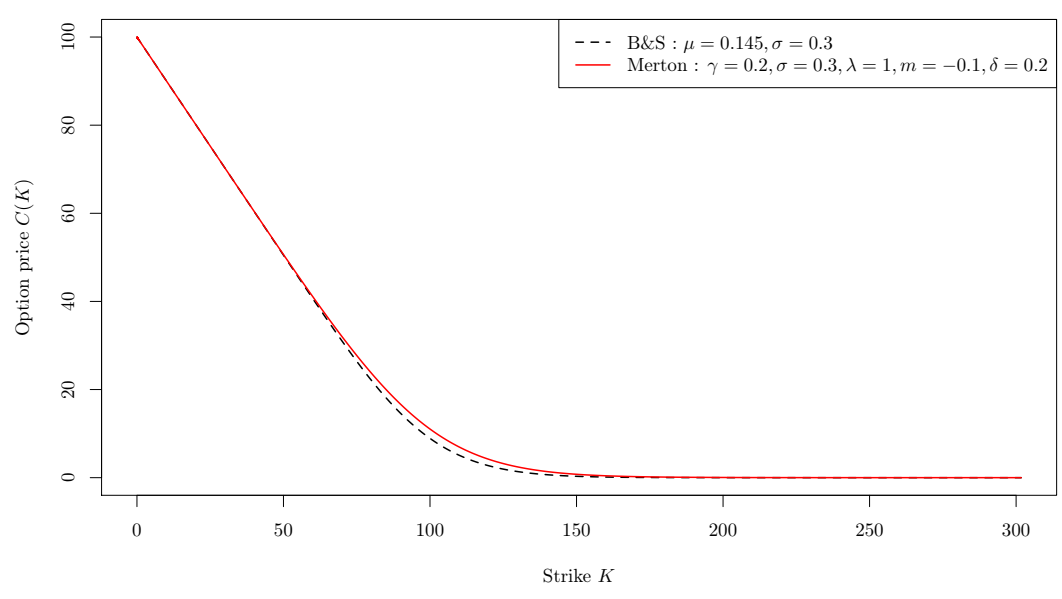

Figure 5: Option price with respect to the strike $K$. A comparison between a Black and Scholes model and a Merton model with the same expectation $\mu-\sigma^{2} / 2=\gamma+\lambda m$.

presented in Figure 6a. For all models, the option price approaches $S_{0}$ as $K \rightarrow 0$ and goes to 0 as $K \rightarrow+\infty$. The jump intensity modifies the option price only for strikes around at-the-money. Otherwise, as the intensity increases, we have more jumps in the period $[0, T]$ and the variance $\operatorname{Var}\left(X_{t}\right)=\left(\sigma^{2}+\lambda\left(m^{2}+\delta^{2}\right)\right) t$ increases. Consequently, the option price increases due to the greater risk that jumps bring to the seller. The same observation is found when the jump size volatility increases. We present it in Figure 6b. The price with respect to the jump size mean is given by Figure 6c. With a negative average of jumps size in the asset price, we have interesting out-the-money options, while in-the-money options are less interesting. A positive average of jumps size leads to reverse statement.

Risk measurement. We compute the VaR and the CVaR of the payoff in the Merton model framework. With the parameters of Table 1 and a strike $K=110$, we illustrate the sensitivity of VaR and CVaR computing with respect to the size of discretization grid in Figures 7 and 8. In Figure 7, we give $\operatorname{VaR}_{a}(H)$ and $\operatorname{CVaR}_{a}(H)$ for different values of $N$, while in Figure 8, we show the absolute variation of the $\mathrm{VaR}$ and the CVaR values with respect to $N$. For the different chosen values of $T$ and $a, \operatorname{VaR}_{a}(H)$ is stable from $N=16384$ and the $\mathrm{CVaR}_{a}(H)$ converges from $N=2048$. The CPU computing time of the method is given in Figure 9. Up to $N=16384$, the algorithm is considered as instantaneous.

\subsection{Variance gamma model}

The variance gamma process is proposed by [33] to describe stock price dynamics istead of the Brownian motion in the original Black and Scholes model. Two new parameters: $m$ skewness and $\kappa$ kurtosis are introduced in order to describe asymmetry and fat tails of real life distributions. A variance gamma process is obtained by evaluating a Brownian motion with a drift at a random time given by a gamma process.

Definition 7.1. The variance gamma $(V G)$ process $\left(Y_{t}\right)_{t \in[0, T]}$ with parameters $(m, \delta, \kappa)$ is defined as

$$
Y_{t}=m \gamma_{t}+\delta B_{\gamma_{t}}
$$




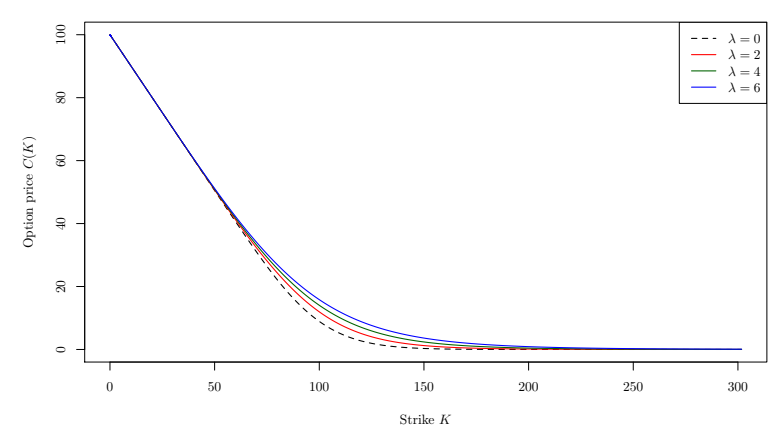

(a) Sensitivity to the jump intensity

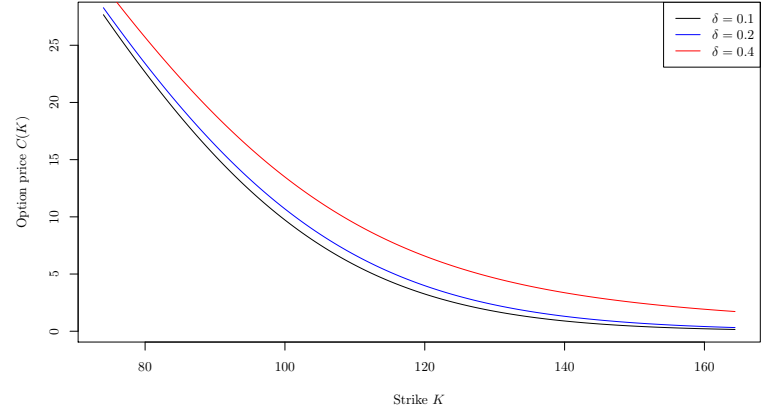

(b) Sensitivity to the jump size variance

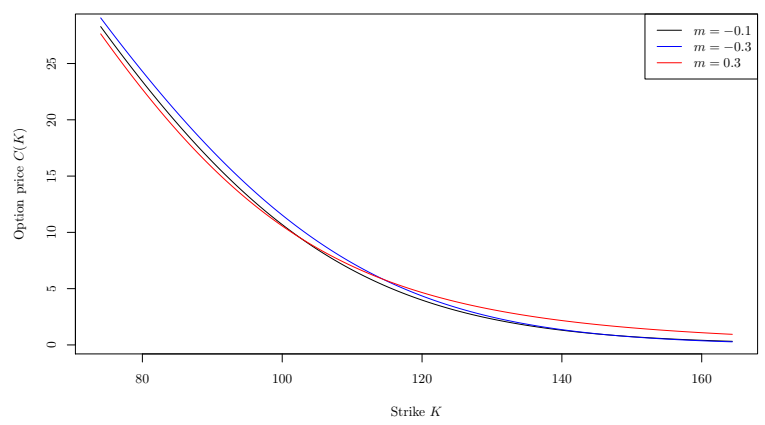

(c) Sensitivity to the jump size mean

Figure 6: Call price sensitivity to the parameters of Merton model.

where $\left(B_{t}\right)_{t \in[0, T]}$ is a standard Brownian motion and $\left(\gamma_{t}\right)_{t \in[0, T]}$ is a gamma process with unit mean rate and variance rate $\kappa$.

Proposition 7.1. The VG process $\left(Y_{t}\right)_{t \in[0, T]}$ is a Lévy process with characteristic function $\Phi_{t}$ and characteristic triplet $\left(\int_{|x| \leqslant 1} x \nu(\mathrm{d} x), 0, \nu\right)$ where

$$
\mathbb{E}\left[e^{i u Y_{t}}\right]=\left(\frac{1}{1-i m \kappa u+\left(\delta^{2} \kappa / 2\right) u^{2}}\right)^{t / \kappa}
$$

and

$$
\nu(x)=\frac{1}{\kappa|x|} \exp \left(\frac{m}{\delta^{2}} x-\frac{\sqrt{\frac{m^{2}}{\delta^{2}}+\frac{2}{\kappa}}}{\delta}|x|\right) .
$$

From the expression of $\nu$, there exists infinitely small jumps. Such a process is called an infinite activity process. It does not admit a distribution of jump size since jumps happen infinitely.

To describe the stock price, we just add a drift component to the VG process. A Brownian component is not necessary and the process moves essentially by jumps. The price process is defined by an exponential Lévy model (1) where

$$
X_{t}=\gamma t+Y_{t}
$$

The process $\left(X_{t}\right)_{t \in[0, T]}$ is a Lévy process with characteristic triplet $\left(\gamma+\int_{|x| \leqslant 1} x \nu(\mathrm{d} x), 0, \nu\right)$ 


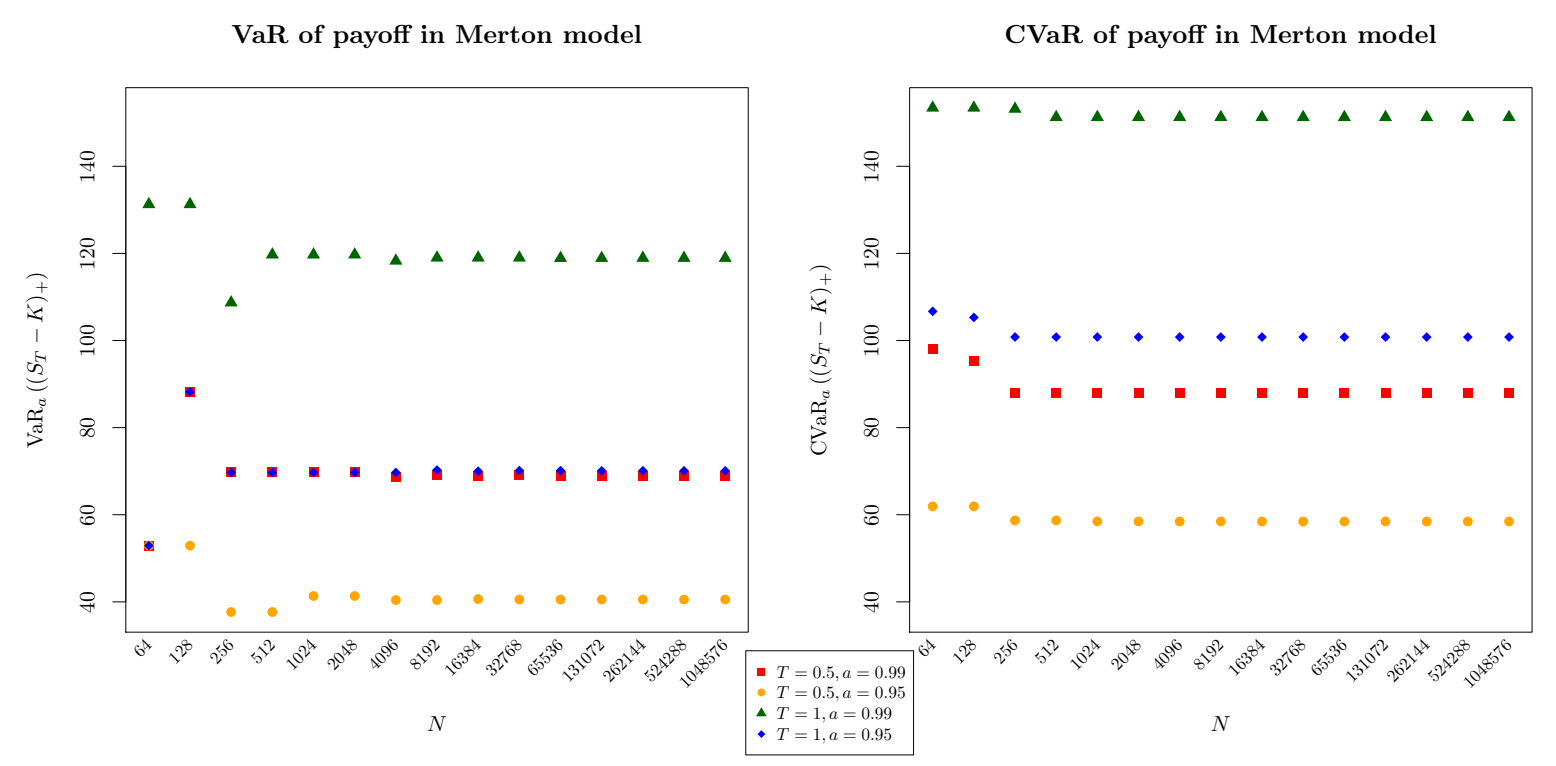

Figure 7: $\operatorname{VaR}_{a}\left(\left(S_{T}-K\right)_{+}\right)$and $\operatorname{CVaR}_{a}\left(\left(S_{T}-K\right)_{+}\right)$with respect to $N$ under a Merton model.

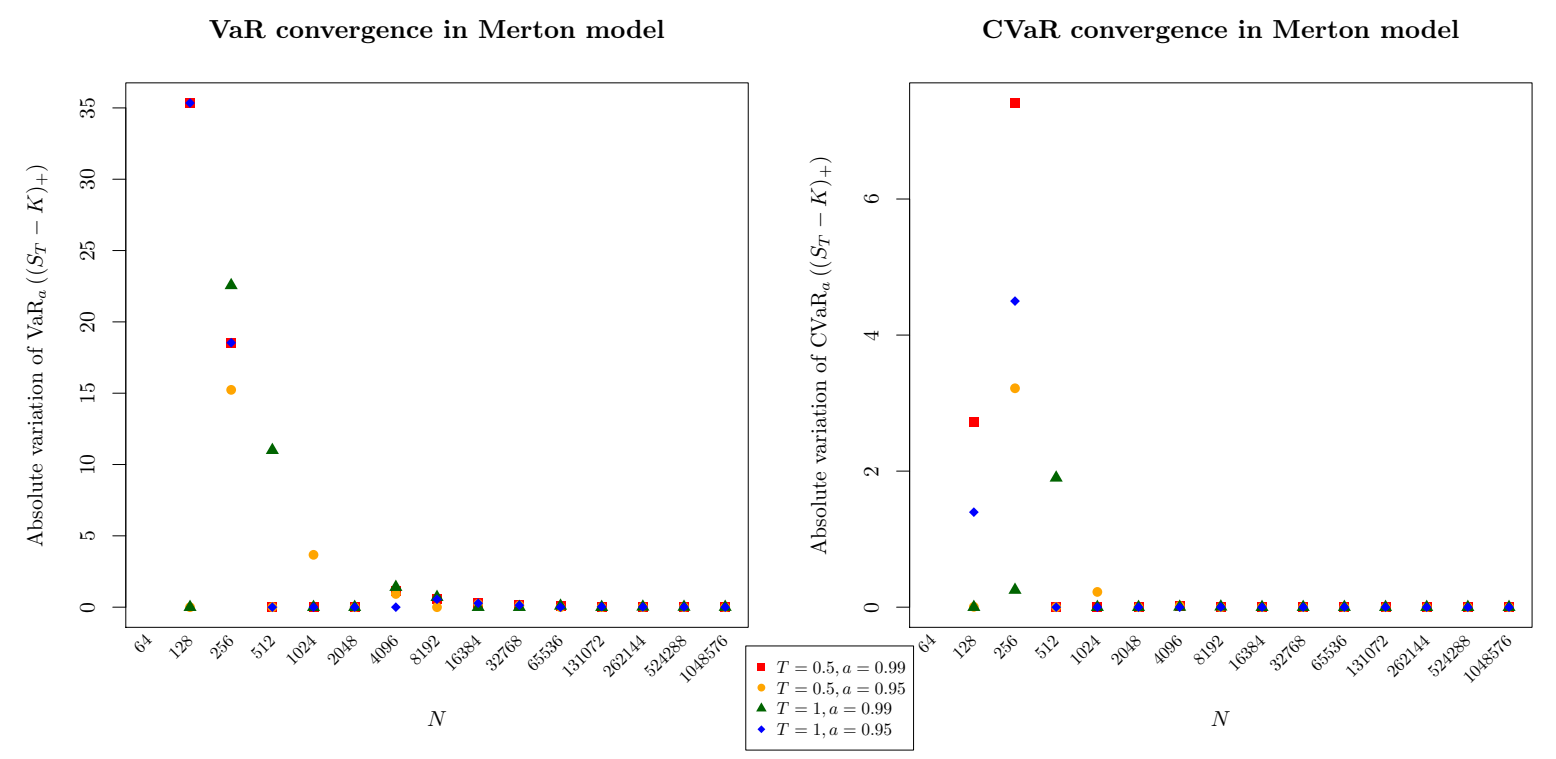

Figure 8: The absolute variation of $\operatorname{VaR}_{a}\left(\left(S_{T}-K\right)_{+}\right)$and $\operatorname{CVaR}_{a}\left(\left(S_{T}-K\right)_{+}\right)$with respect to $N$ under a Merton model. 


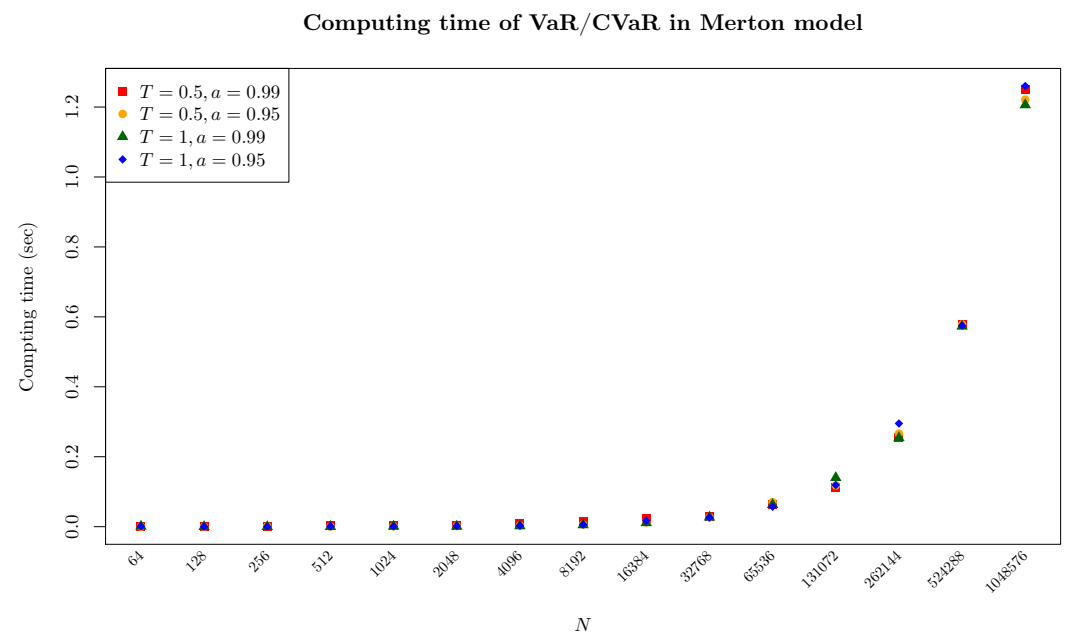

Figure 9: CPU time of the VaR/CVaR computing method under a Merton model.

and characteristic function given by

$$
\Phi_{t}(u)=\mathbb{E}\left[e^{i u X_{t}}\right]=\frac{e^{i \gamma u t}}{\left(1-i m \kappa u+\left(\delta^{2} \kappa / 2\right) u^{2}\right)^{t / \kappa}} .
$$

The procedure to fit the variance gamma model to financial data is explained in [45].

Esscher martingale measure. By solving the inequality $1-i m \kappa u+\left(\delta^{2} \kappa / 2\right) u^{2}>0$ with respect to $u$, we show that $X_{t}$ for some $t$ or, equivalently, for all $t$, possesses a moment generating function $u \mapsto \mathbb{E}\left[\exp \left(u X_{t}\right)\right]$ on

$$
\left(-m / \delta^{2}-\sqrt{m^{2} / \delta^{2}+2 / \kappa} \delta,-m / \delta^{2}+\sqrt{m^{2} / \delta^{2}+2 / \kappa} \delta\right) .
$$

To apply Theorem 3.1, we assume that $2 \sqrt{m^{2} / \delta^{2}+2 / \kappa} \delta>1$. Under this assumption, we look for $\theta \in\left(-m / \delta^{2}-\sqrt{m^{2} / \delta^{2}+2 / \kappa} \delta,-m / \delta^{2}+\sqrt{m^{2} / \delta^{2}+2 / \kappa} \delta-1\right)$ solution to

$$
\mathbb{E}\left[e^{(\theta+1) X_{t}}\right]=e^{r t} \mathbb{E}\left[e^{\theta X_{t}}\right] .
$$

For the parameters given in Table 1, the moment generating function of $X_{t}$ is well defined on $(-3.15,3.17)$. Numerically, we obtain $\theta=-0.57$.

The characteristic function of $\left(X_{t}\right)_{t \in[0, T]}$ under $\mathbb{P}^{*}$ is given by

$$
\Phi_{t}^{*}(u)=\frac{\mathbb{E}\left[e^{(\theta+i u) X_{t}}\right]}{\mathbb{E}\left[e^{\theta X_{t}}\right]}=\frac{e^{i \gamma u t}}{\left(1-i m^{*} \kappa u+\left(\delta^{*} \kappa / 2\right) u^{2}\right)^{t / \kappa}},
$$

where $m^{*}=\left(m+\delta^{2} \theta\right) / A, \delta^{*}=\delta / \sqrt{A}$ and $A=1-m \kappa \theta-\frac{\delta^{2} \kappa}{2} \theta^{2}$. Hence, $\left(X_{t}\right)_{t \in[0, T]}$ is once again a variance gamma process under $\mathbb{P}^{*}$ with characteristic triplet $\left(\gamma+\int_{|x| \leqslant 1} x \nu^{*}(\mathrm{~d} x), 0, \nu^{*}\right)$ where

$$
\nu^{*}(x)=e^{\theta x} \nu(x)=\frac{1}{\kappa|x|} \exp \left(\frac{m^{*}}{\delta^{*^{2}}} x-\frac{\sqrt{\frac{m^{*^{2}}}{\delta^{2}}+\frac{2}{\kappa}}}{\delta^{*}}|x|\right) .
$$

The initial Lévy density and the risk-neutral Lévy density are given in Figure 10. With our choice of parameters, the general trend $\mathbb{E}\left[X_{t}\right]=(\gamma+m) t$ is positive, which leads to a negative $\theta$. The large positive jumps will be nearly irrelevant for pricing options, while large negative jumps will still contribute. 


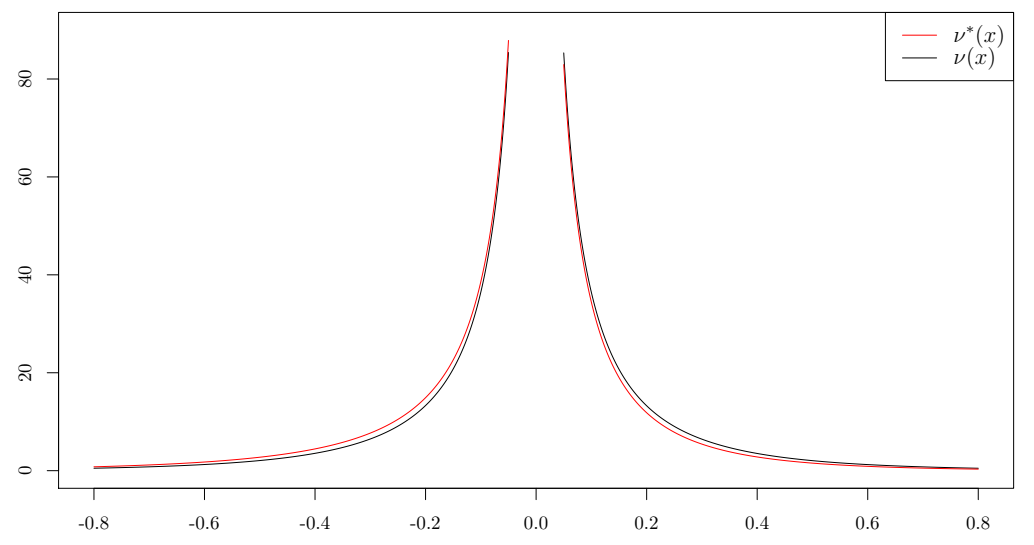

Figure 10: The risk neutral Lévy density $\nu^{*}(x)$ of the Esscher martingale measure in the variance gamma model, compared to the initial Lévy density $\nu(x)$.

Pricing with FFT under the Esscher martingale measure. The explicit form of the characteristic function under the Esscher martingale measure $\mathbb{P}^{*}$ is applied to price call options. Figures $11 \mathrm{a}$ and $11 \mathrm{~b}$ represent the sensitivity of the call price to a variation of the parameters $\delta$ and $\kappa$ respectively. We take the parameters of Table 1 except for $\delta$ that varies in $\{0.6,0.8,1\}$ in Figure $11 \mathrm{a}$ and $\kappa$ that varies in $\{0.02,0.2\}$ in Figure $11 \mathrm{~b}$. The parameters $\delta$ and $\kappa$ provide control over volatility and kurtosis respectively. Increasing $\delta$ leads to greater volatility which in turn increases the option price. On the other hand, when $\kappa$ inscreases, the distribution tails of the asset price become fatter and the price of the out-the-money call increases, while the price of the at-the-money option decreases.

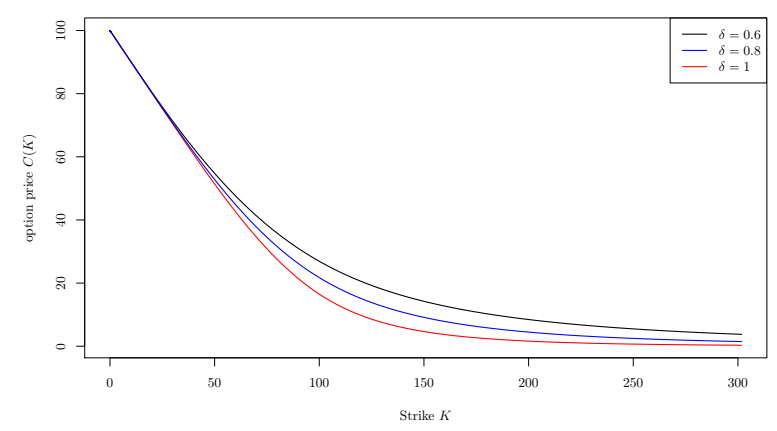

(a) Sensitivity to the volatility parameter

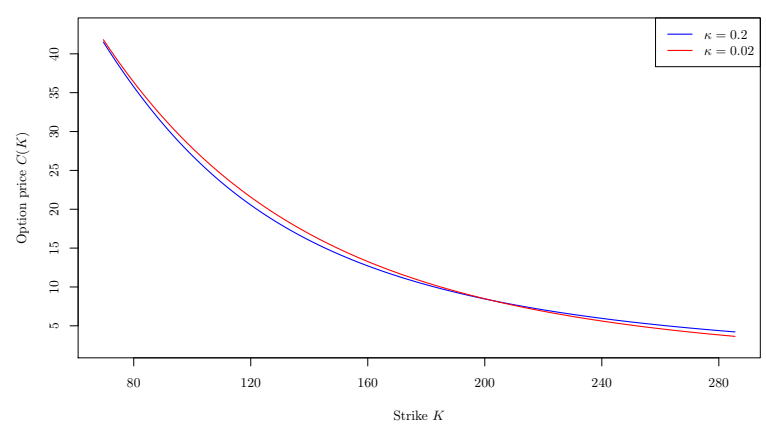

(b) Sensitivity to the kurtosis parameter

Figure 11: Call price sensitivity to the parameters of the variance gamma model.

Risk measurement. We compute the VaR and the CVaR of the payoff under a variance gamma model. With the parameters of Table 1 and a strike $K=110$, we illustrate the sensitivity of $\mathrm{VaR}$ and $\mathrm{CVaR}$ computing with respect to the size of discretization grid in Figures 12 and 13. In Figure 12, we give $\operatorname{VaR}_{a}(H)$ and $\operatorname{CVaR}_{a}(H)$ for different values of $N$, while in Figure 13 , we show the absolute variation of the $\mathrm{VaR}$ and the CVaR values with respect to $N$. For different values of $T$ and $a, \operatorname{VaR}_{a}(H)$ is stable from $N=16384$ and the $\operatorname{CVaR}_{a}(H)$ converges from $N=1024$. The CPU computing time of the method 


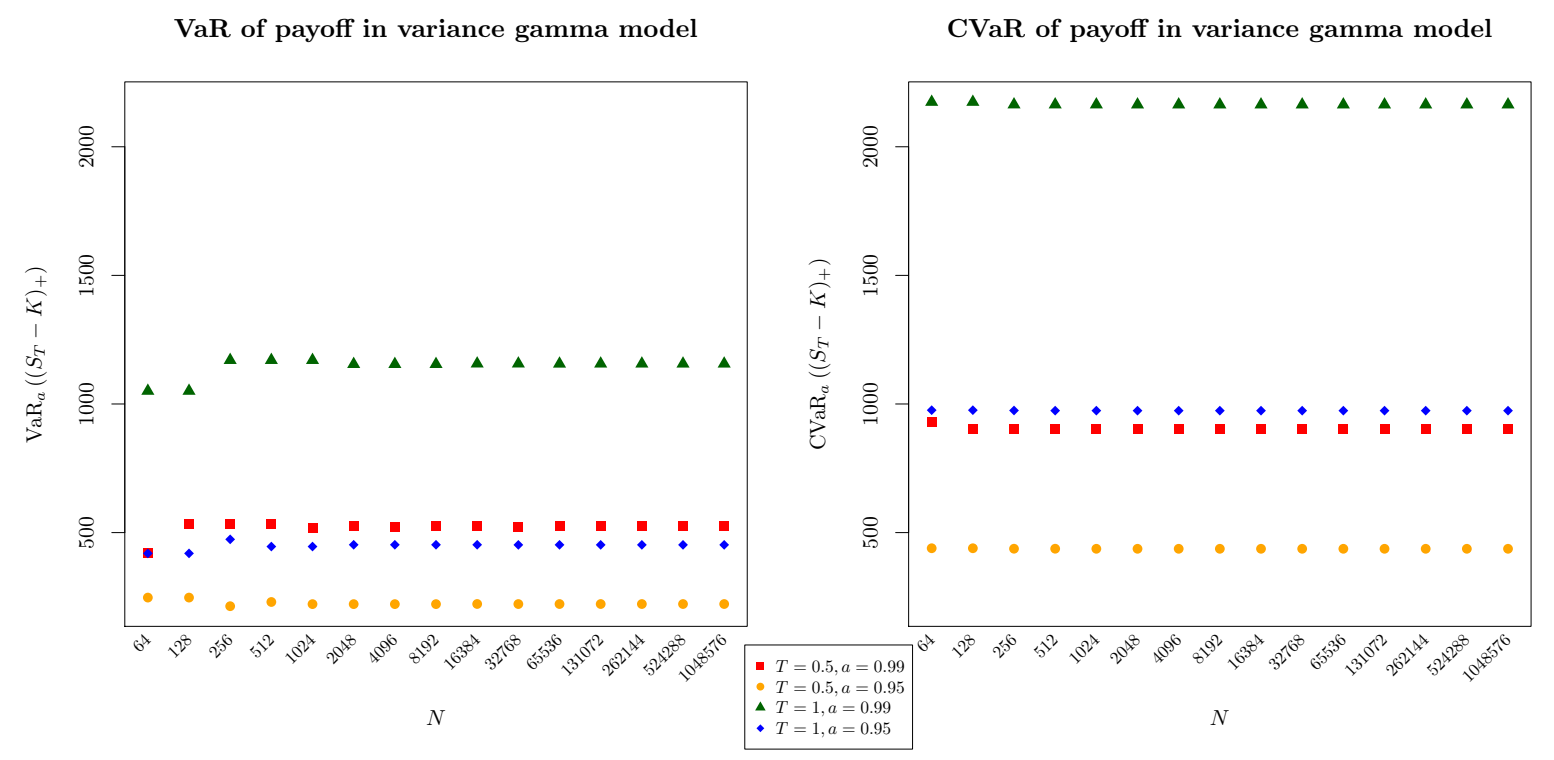

Figure 12: $\operatorname{VaR}_{a}\left(\left(S_{T}-K\right)_{+}\right)$and $\mathrm{CVaR}_{a}\left(\left(S_{T}-K\right)_{+}\right)$with respect to $N$ under a variance gamma model.

is given in Figure 14. Up to $N=16384$, the algorithm is considered as instantaneous.

We conclude that for the three models, the value $N=16384$ permits to give a stable value of both $\operatorname{VaR}_{a}(H)$ and $\mathrm{CVaR}_{a}(H)$, with a negligible computing time.

\section{Conclusion}

In this survey, we studied step by step how to price European option under an Exponential Lévy model. We used the Esscher transform technique to construct two equivalent martingale measures: the Esscher martingale measure and the minimal entropy martingale measure. From the observation that the compound return and the simple return of a stock price process are very close, we showed that these two measures are almost similar. However, the minimal entropy martingale measure is not adequate to the Carr-Madan pricing method as the characteristic function under this latter does not have an analytic expression, even in simple models. Under the Esscher martingale measure, we numerically computed the price of European option using the Carr-Madan method based on the fast Fourier transform. Numerical error of the Carr-Madan method is shown with comparison to the closed-form formula in the Black and Scholes context. Moreover, the sensitivity of option price to jumps is presented in the Merton model and the variance gamma model with respect to jump parameters. Finally, applications of Fourier inversion technique in Risk measurement are proposed to compute the VaR and the CVaR of derivatives, as well as the VaR and the CVaR of monetary loss and loss in terms of returns.

\section{References}

[1] C. Acerbi and D. Tasche. On the coherence of expected shortfall. Journal of Banking \& Finance, 26(7):1487-1503, 2002.

[2] D. Applebaum. Lévy processes and stochastic calculus. Cambridge University Press, 2009. 


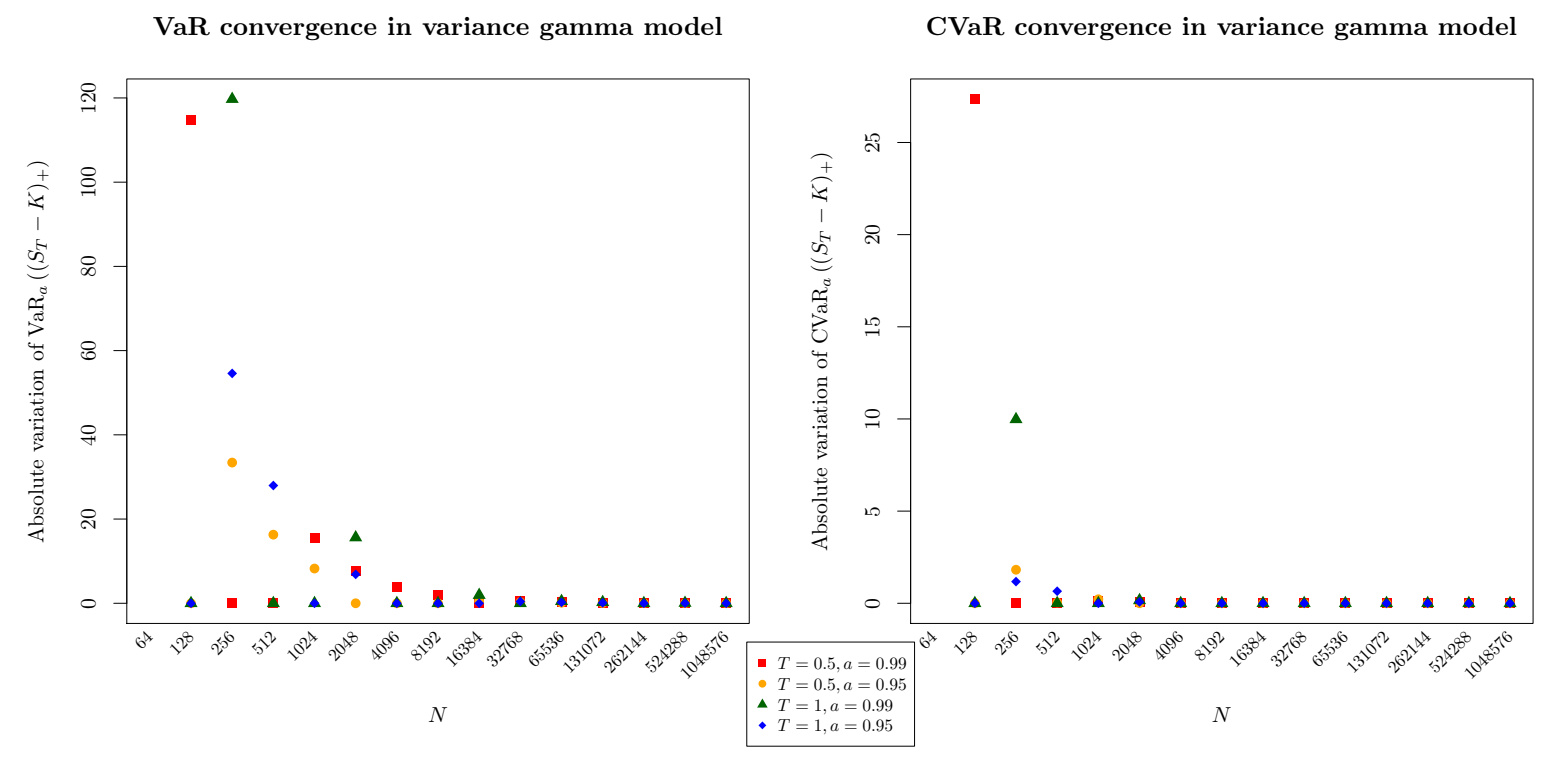

Figure 13: The absolute variation of $\operatorname{VaR}_{a}\left(\left(S_{T}-K\right)_{+}\right)$and $\operatorname{CVaR}_{a}\left(\left(S_{T}-K\right)_{+}\right)$with respect to $N$ under a variance gamma model.

Computing time of VaR/CVaR in variance gamma model

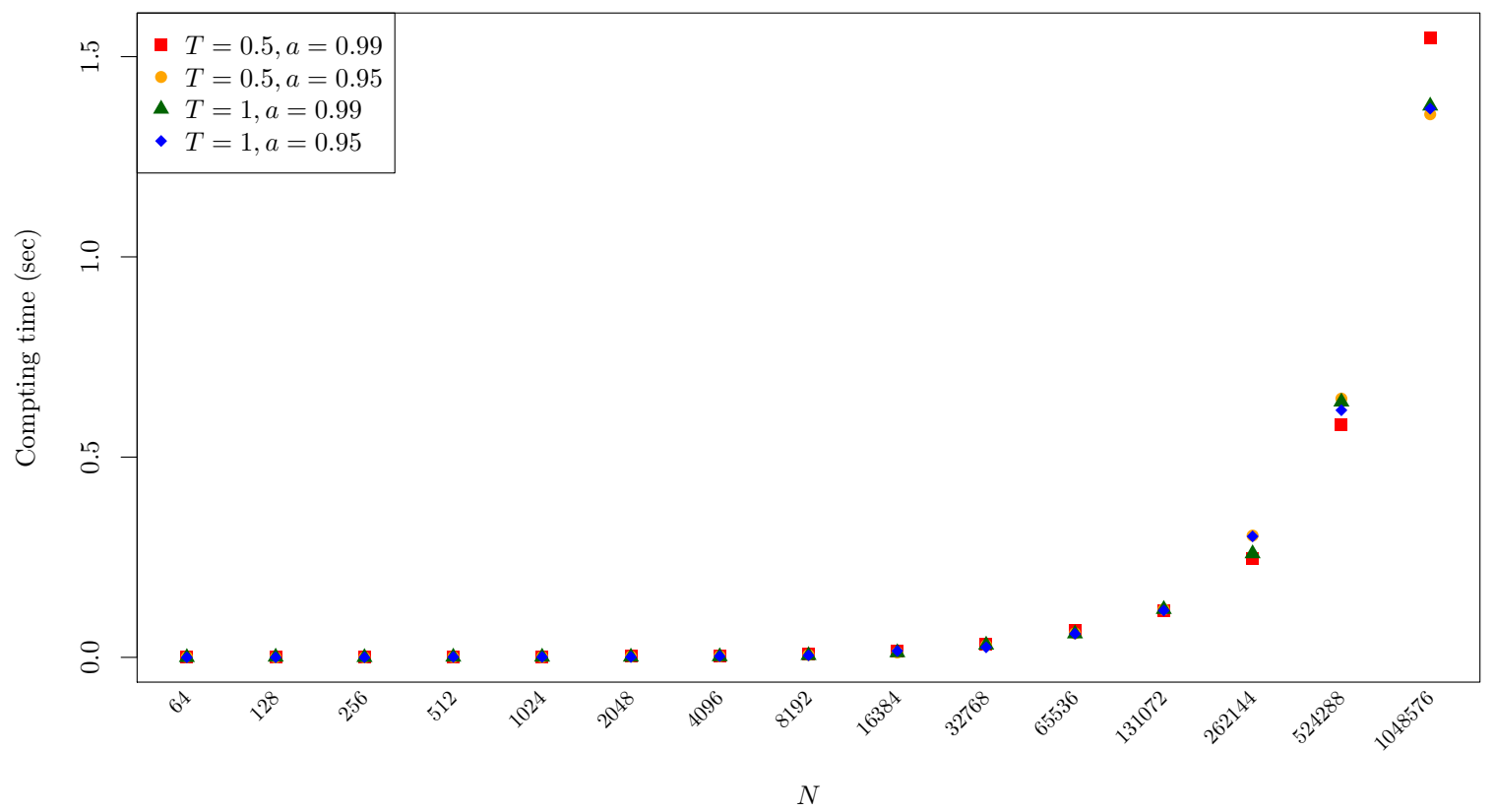

Figure 14: CPU time of the $\mathrm{VaR} / \mathrm{CVaR}$ computing method under a variance gamma model. 
[3] O. E. Barndorff-Nielsen. Normal inverse Gaussian distributions and stochastic volatility modelling. Scandinavian Journal of Statistics, 24(1):1-13, 1997.

[4] O. E. Barndorff-Nielsen. Processes of normal inverse Gaussian type. Finance and Stochastics, 2(1):41-68, 1997.

[5] D. S. Bates. Post-crash moneyness biases in S\&P 500 futures options. Rodney, L. White Center working paper, Wharton School, University of Pennsylvania, Philadelpia, PA, 1995.

[6] F. E. Benth, M. Groth, and P. C. Kettler. A quasi-Monte Carlo algorithm for the normal inverse Gaussian distribution and valuation of financial derivatives. International Journal of Theoretical and Applied Finance, 9(06):843-867, 2006.

[7] J. Bertoin. Lévy processes, volume 121. Cambridge University Press, 1998.

[8] F. Black and M. Scholes. The pricing of options and corporate liabilities. Journal of Political Economy, 81(3):637-654, 1973.

[9] H. Bühlmann, F. Delbaen, P. Embrechts, and A. N. Shiryaev. No-arbitrage, change of measure and conditional Esscher transforms. CWI quarterly, 9(4):291-317, 1996.

[10] P. Carr, H. Geman, D. Madan, and M. Yor. The fine structure of asset returns: An empirical investigation. The Journal of Business, 75(2):305-333, 2002.

[11] P. Carr and D. Madan. Option valuation using the fast Fourier transform. Journal of Computational Finance, 2(4):61-73, 1999.

[12] T. Chan. Pricing contingent claims on stocks driven by Lévy processes. Annals of Applied Probability, 9(2):504-528, 1999.

[13] R. Cont and P. Tankov. Financial modelling with jump processes. Chapman \& Hall/CRC Financial Mathematics Series. Chapman \& Hall/CRC, Boca Raton (Fla.), London, New York, 2004.

[14] J. W. Cooley and J. W. Tukey. An algorithm for the machine calculation of complex Fourier series. Mathematics of Computation, 19(90):297-301, 1965.

[15] E. Eberlein and U. Keller. Hyperbolic distributions in finance. Bernoulli, 1(3):281299, 1995.

[16] E. Eberlein, U. Keller, and K. Prause. New insights into smile, mispricing, and Valueat-Risk: The hyperbolic model. The Journal of Business, 71(3):371-405, 1998.

[17] R. J. Elliott, L. Chan, and T. K. Siu. Option pricing and Esscher transform under regime switching. Annals of Finance, 1(4):423-432, 2005.

[18] H. Follmer and M. Schweizer. Hedging of contingent claims. Applied Stochastic Analysis, 5:389, 1991.

[19] M. Frittelli. The minimal entropy martingale measure and the valuation problem in incomplete markets. Mathematical Finance, 10(1):39-52, 2000. 
[20] T. Fujiwara and Y. Miyahara. The minimal entropy martingale measures for geometric Lévy processes. Finance and Stochastics, 7(4):509-531, 2003.

[21] H. Geman. Pure jump Lévy processes for asset price modelling. Journal of Banking E Finance, 26(7):1297-1316, 2002.

[22] H.-U. Gerber and E. S. W. Shiu. Option pricing by Esscher transforms. Transactions of the Society of Actuaries, XLVI:99-191, 1994.

[23] P. Glasserman. Monte Carlo methods in financial engineering, volume 53. Springer Science \& Business Media, 2003.

[24] T. Goll and L. Rüschendorf. Minimax and minimal distance martingale measures and their relationship to portfolio optimization. Finance and Stochastics, 5(4):557-581, 2001.

[25] S. L. Heston. A closed-form solution for options with stochastic volatility with applications to bond and currency options. Review of Financial Studies, 6(2):327-343, 1993.

[26] F. Hubalek and C. Sgarra. Esscher transforms and the minimal entropy martingale measure for exponential Lévy models. Quantitative Finance, 6(2):125-145, 2006.

[27] J. Hugonnier, D. Kramkov, and W. Schachermayer. On utility-based pricing of contingent claims in incomplete markets. Mathematical Finance, 15(2):203-212, 2005.

[28] J. Jacod and A. Shiryaev. Limit theorems for stochastic processes, volume 288. Springer Science \& Business Media, 2013.

[29] P. Jorion. Value at Risk: The New Benchmark for Managing Financial Risk. McGraw-Hill, 3 edition, 2006.

[30] S. G. Kou. A jump-diffusion model for option pricing. Management Science, 48(8):1086-1101, 2002.

[31] R. W. Lee et al. Option pricing by transform methods: extensions, unification and error control. Journal of Computational Finance, 7(3):51-86, 2004.

[32] D. Madan, P. Carr, and E. Chang. The variance gamma process and option pricing. European Finance Review, 2(1):79-105, 1998.

[33] D. Madan and E. Seneta. The variance gamma (V.G.) model for share market returns. The Journal of Business, 63(4):511-524, 1990.

[34] R. C. Merton. Option pricing when underlying stock returns are discontinuous. Journal of Financial Economics, 3(1-2):125-144, 1976.

[35] Y. Miyahara. Canonical martingale measures of incomplete assets markets. Probability Theory and Mathematical Statistics (Tokyo, 1995), pages 343-352, 1996.

[36] Y. Miyahara. Geometric Lévy Process \& MEMM: pricing model and related estimation problems. Asia-Pacific Financial Markets, 8(1):45-60, 2001. 
[37] R. T. Rockafellar and S. Uryasev. Optimization of Conditional Value-at-Risk. Journal of Risk, 2:21-41, 2000.

[38] R. T. Rockafellar and S. Uryasev. Conditional Value-at-Risk for general loss distributions. Journal of Banking and Finance, 26(7):1443-1471, 2002.

[39] M. Rubinstein. Nonparametric tests of alternative option pricing models using all reported trades and quotes on the 30 most active CBOE option classes from august 23, 1976 through august 31, 1978. The Journal of Finance, 40(2):455-480, 1985.

[40] M. Rubinstein. Implied binomial trees. The Journal of Finance, 49(3):771-818, 1994.

[41] T. H. Rydberg. The normal inverse Gaussian Lévy process: simulation and approximation. Communications in Statistics. Stochastic Models, 13(4):887-910, 1997.

[42] K. Salhi. Financial extreme risks : analysis and modeling. PhD thesis, University of Lorraine, 2016.

[43] K.-i. Sato. Lévy processes and infinitely divisible distributions. Cambridge University Press, 1999.

[44] M. Schweizer. On the minimal martingale measure and the Föllmer-Schweizer decomposition. Stochastic Analysis and Applications, 13(5):573-599, 1995.

[45] E. Seneta. Fitting the variance-gamma model to financial data. Journal of Applied Probability, 41:177-187, 2004.

[46] D. Synowiec. Jump-diffusion models with constant parameters for financial log-return processes. Computers \& Mathematics with Applications, 56(8):2120-2127, 2008.

[47] J. S. Walker. Fast Fourier transforms, volume 24. CRC press, 1996.

[48] P. Wilmott. Derivatives, the theory and practice of financial engineering. John Wiley\&SSons, Chichester, 1998.

[49] L. Yao, G. Yang, and X. Yang. A note on the mean correcting martingale measure for geometric Lévy processes. Applied Mathematics Letters, 24(5):593-597, 2011.

[50] J. Zhu. Modular Pricing of Options (MPO). In Modular Pricing of Options: An Application of Fourier Analysis, pages 25-97. Springer, 2000. 